\title{
Computer-Assisted Design of Macrocyclic Chelators for Actinium-225 Radiotherapeutics
}

\author{
Amanda Morgenstern, ${ }^{\text {ta }}$ Laura M. Lilley, ${ }^{\mathrm{b}}$ Benjamin W. Stein, ${ }^{\mathrm{b}}$ Stosh A. \\ Kozimor, ${ }^{* b}$ Enrique R. Batista, ${ }^{* a}$ and Ping Yang ${ }^{* a}$ \\ Supplementary Information
}

S1. Computational Details....................................................... 2

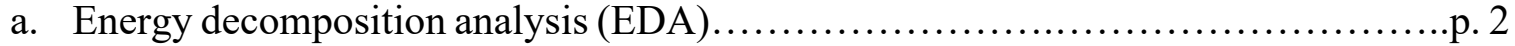

b. Natural orbitals of chemical valence (NOCV) ................................ 3

c. Quantum theory of atoms in molecules (QTAIM) ........................... 4

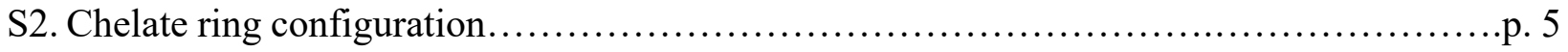

S3. Ring configuration of Ac-complexes............................................... 6

S4. Alternative Ac(HEHA) ${ }^{3-}$ configurations........................................... 8

S5. Definition of atomic planes................................................... 9

S6. QTAIM analysis of free ligands and $\mathrm{Ac}^{3+}$ complexes............................... 10

S7. Mulliken charges................................................................ 12

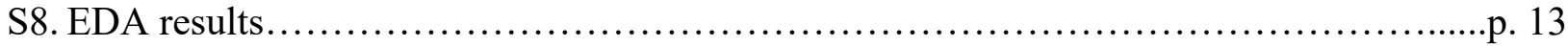

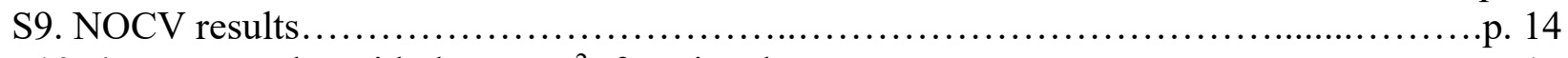

$\mathrm{S} 10.4 \mathrm{~N}-$ macrocyles with three $\mathrm{PO}_{3}{ }^{2-}$ functional groups ............................... 17

S11. Ac-Complex Coordinates................................................... 18

References......................................................................... 33

a. Theoretical Division, Los Alamos National Laboratory, Los Alamos, NM 87545, USA. E-mail: pyang@lanl.gov and erb@lanl.gov

b. Chemistry Division, Los Alamos National Laboratory, Los Alamos, NM 87545, USA. E-mail: stosh@lanl.gov

${ }^{\dagger}$ Author's current address: Chemistry and Biochemistry Department, University of Colorado at Colorado Springs, Colorado Springs, CO 80918, USA 


\section{S1. Computational Details}

Previous experimental work to determine structures and binding constants was performed in basic conditions, such that all compounds were fully deprotonated. ${ }^{1}$ Basic buffers should be effective in the radiolabeling process since $\mathrm{Ac}^{3+}$ is the most basic +3 ion on the periodic table and its first hydrolysis constant is $9.4,{ }^{2,3}$ lowering the amount of $\mathrm{AcOH}^{+}$produced.

Computational tests on the effect of protonation state on binding energies showed that chelator binding improved inversely to the number of protons on the chelator. A simple test on the effect of protonation on the binding ability of $\mathrm{DOTP}^{8-}$ is shown in Table $\mathrm{S} 1$. For this study, we chose to run all simulations with fully deprotonated chelators.

\begin{tabular}{|l|r|r|}
\hline Protons (n) & Charge (n-5) & $\boldsymbol{\Delta} \mathbf{E ~ ( k c a l / m o l ) ~}$ \\
\hline 0 & -5 & -27.9 \\
\hline 1 & -4 & -19.0 \\
\hline 2 & -3 & -8.7 \\
\hline 3 & -2 & +1.1 \\
\hline 4 & -1 & +14.5 \\
\hline
\end{tabular}

Table S1: $\triangle E$ for the reaction, $A c(D O T A)^{-}+H_{n}$ DOTP ${ }^{n-8} \rightarrow A c\left(H_{n} D O T P\right)^{n-5}+D^{2} A^{4-} . H_{n} D O T P^{n-8}$ is less favorable than DOTA ${ }^{4-}$ for $A c^{3+}$ chelation when $n>3$.

For radiolabeling, $\mathrm{Ac}^{3+}$ is originally in an aqueous solution and undergoes the reaction,

$$
(\mathrm{S} 1) \mathrm{Ac}\left(\mathrm{H}_{2} \mathrm{O}\right)_{\mathrm{m}}{ }^{3+}+\mathrm{L}^{\mathrm{n}-} \longrightarrow \mathrm{Ac}(\mathrm{L})^{3-\mathrm{n}}+\mathrm{m}\left(\mathrm{H}_{2} \mathrm{O}\right)
$$

where $\mathrm{L}^{\mathrm{n}-}$ is the chelating ligand. There are multiple sources of uncertainty in this model, including the number of coordinating water molecules to actinium (m), the bonding network of bulk solvent with coordinating water molecules, and the bonding network of the coordinating water itself. To minimize errors, we instead use the ligand exchange reaction presented in the main text, Eqn. (1), which compares all chelating agents to the current state-of-the-art chelator, DOTA $^{4-}$.

Due to the closed-shell configuration of $\mathrm{Ac}^{3+}$ complexes, the size of the frozen core had a negligible effect on geometric parameters. To demonstrate, geometry optimizations were run for Ac(DOTA) $)^{-}$using the PBE functional, TZP basis set, and the same COSMO and ZORA parameters denoted in the main text. Optimization using large, small, and no frozen core gave average ( \pm standard deviations) Ac-O bond distances of $2.549 \pm 0.001,2.545 \pm 0.002$, and 2.550 $\pm 0.001 \AA$, and Ac-N bond distances of $2.844 \pm 0.002,2.844 \pm 0.002$, and $2.840 \pm 0.002 \AA$, 
respectively. This is a range of only $0.005 \AA$ for average Ac-O bond distances and a range of $0.004 \AA$ for average Ac-N bond distances.

\section{a. Energy Decomposition Analysis (EDA):}

There are a multitude of methods for partitioning the total bonding energy between two fragments into contributions from specific interactions, such as Pauli repulsion, orbital overlap, etc. We report on the Morokuma scheme of energy decomposition method as implemented in ADF. ${ }^{4-6}$ The chelator molecule was defined as one of the fragments and the actinium ion as the other. The total bond energy, $\Delta E$, is decomposed as

$$
\text { (S2) } \Delta E=\Delta E_{\mathrm{prep}}+\Delta E_{\mathrm{int}}
$$

where $\Delta E_{\text {prep }}$ is the amount of energy required to deform the separated fragments, which considers both geometry changes and any excitations of electrons required for the valence electronic configuration. $\Delta E_{\text {int }}$ is the energy due to interaction between the fragments, which can be further decomposed into

$$
\text { (S3) } \Delta E_{\text {int }}=\Delta V_{\text {elst }}+\Delta E_{\text {Pauli }}+\Delta E_{\text {oi }}
$$

$\Delta V_{\text {elst }}$ is the energy classical electrostatic energy from overlapping the fragment densities. $\Delta E_{\text {Pauli }}$ corresponds to the Pauli repulsions that occurs from antisymmetrization and renormalization of the product of fragment wavefunctions. Finally, the orbital interaction term, $\Delta E_{\mathrm{oi}}$ is the energy associated with orbital relaxation from electron pair bonding, charge transfer, and polarization. In general, $\Delta V_{\text {elst }}$ and $\Delta E_{\mathrm{oi}}$ are negative, while $\Delta E_{\text {Pauli }}$ is a repulsive, positive energy. These energy terms can be used to quantify how ionic/covalent the bonding is between fragments using,

$$
\begin{gathered}
(S 4) \% \text { Ionic }=\left(\Delta V_{\text {elst }}+\Delta E_{\text {Pauli }}\right) /\left(\Delta V_{\text {elst }}+\Delta E_{\text {Pauli }}+\Delta E_{\text {oi }}\right) \\
\text { (S5) \%Covalent }=\left(\Delta E_{\text {oi }}\right) /\left(\Delta V_{\text {elst }}+\Delta E_{\text {Pauli }}+\Delta E_{o i}\right)
\end{gathered}
$$

$\Delta E_{\mathrm{oi}}$ can be further analyzed using the NOCV method described below.

\section{b. Natural Orbitals of Chemical Valence (NOCV):}

The NOCV method is used to analyze chemical bonding by decomposing the deformation density, $\Delta \rho$, into orbital contributions. ${ }^{7}$ The deformation density is defined as the change in the charge density of the fragments in their final geometry and electronic state when calculated separately, and when the fragments are allowed to interact. $\Delta \rho$ thus shows the flow of 
electrons as the chelator binds to the actinium ion. Each $N O C V \Psi_{i}$ is an eigenvector of the deformation density matrix,

$$
\text { (S6) } \Delta P \Psi=v_{i} \Psi_{i}
$$

The deformation density can be expressed in the NOCV representation as a sum of pairs of complimentary eigenfunctions $\left(\psi-k, \psi_{k}\right)$ corresponding to eigenvalues $-v_{\mathrm{k}}$ and $v_{\mathrm{k}}$ with the same absolute value but opposite signs. The eigenvalues of the NOCVs give the energy of each orbital, and the sum of these energies gives the total orbital component of the energy decomposition $\left(\Delta E_{\mathrm{oi}}\right)$.

$$
\text { (S7) } \Delta \rho(r)=\sum \Delta \rho_{k}(r)=\sum v_{k}\left(-\Psi^{2}{ }_{-k}(r)+\Psi_{k}^{2}(r)\right)
$$

\section{c. Quantum Theory of Atoms in Molecules (QTAIM):}

The standard Bader analysis package in $\mathrm{ADF}^{8,9}$ was used to analyze the charge density in complexed systems. QTAIM classifies charge density in terms of topological structure. ${ }^{10}$ Critical points (CPs), i.e. points where the gradient of the charge density vanishes, can be used to determine structural and reactivity information. Bond CPs are saddle points in the charge density that exist between bonded nuclear (maxima) CPs. The magnitude of the charge density at bond CPs has been found to correlate with bond strength. ${ }^{11,12}$ Ridges of charge density (bond paths) are present between bond and nuclear CPs wherever a topological bonding interaction exists. QTAIM also provides an unambiguous approach to partitioning a molecule into its atoms or atomic basins. Integrating the number of electrons in an atomic basin provides the Bader atomic charge. 


\section{S2. Chelate ring configuration}

Select chelate ring angles in Ac(DOTA) $)^{-}$are shown in Table S2. All angles are more similar to a 5-membered ring than 6-membered ring (with the same $\mathrm{C} / \mathrm{N} / \mathrm{O}$ atom types). Figure $\mathrm{S} 1$ shows a side view of the macrocycle chelate ring forms with the macrocycle in $\operatorname{Ac}(\mathrm{DOTA})^{-}$. This 5membered chelate ring has a chair geometry, which is also found in the 6-membered hydrocarbon ring, cyclohexane.

\begin{tabular}{|l|l|l|l|l|}
\hline & Macrocycle N-C-C & Macrocycle C-N-C & Arm N-C-C & Arm C-C-O \\
\hline Ac(DOTA) & 113.6 & 111.0 & 114.0 & 117.0 \\
\hline 5-membered ring & 107.0 & 104.2 & 107.0 & 106.1 \\
\hline 6-membered ring & 114.4 & 111.7 & 114.4 & 112.1 \\
\hline
\end{tabular}

Table S2: Angles ( $\left.{ }^{\circ}\right)$ in Ac(4N-macrocycle)- complexes compared to 5-and 6-membered rings with equivalent number of $C$, $N$, and $O$ atoms.
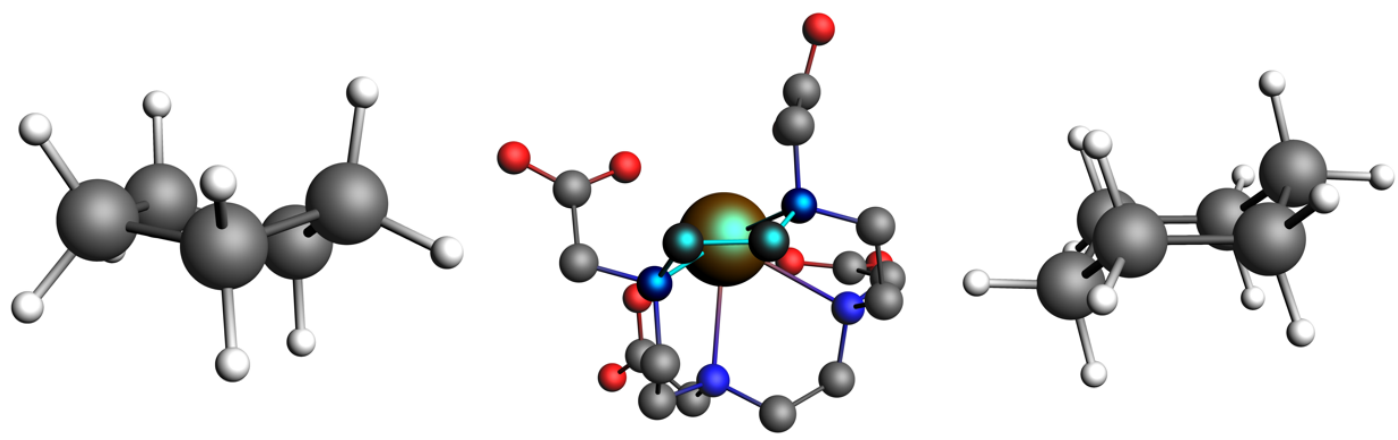

Figure S1: Geometry of five-membered rings created with actinium (highlighted atoms in center image correspond to macrocycle ring) are more similar to a six-membered carbon ring (right) than a five-membered ring (left), due to the large size of the actinium ion, which takes up almost as much space as two carbon atoms. 


\section{S3. Ring configuration of Ac-complexes}

While DOTA ${ }^{4-}$ binds metals in a traditional encapsulated coordination structure, metals can have an alternative encircled geometry, where the metal is placed inside a macrocyclic ring with the pendant acetate arms alternating wrapping above and below the ring as shown in Figure S2. Alternative encircled geometries were tested with biological applications in mind, as the alternating arm positions will disperse the negative charges on the functional groups more evenly around the actinium, leading to an overall complex that is less polar. For the larger macrocycles in this study, such as $\mathrm{HEHA}^{6-}$, multiple configurations for the arms can result in favorable metal coordination, e.g. three up, three down or four up, two down. Based on NMR studies, the tetravalent actinide Th(IV) is thought to complex with HEHA in an encircled geometry with three arms up and three arms down, in an alternating pattern for the thermodynamically stable complex. The kinetically stable isomer of Th(HEHA $)^{2-}$ however, was believed to have two acetate arms above the nitrogen ring and four acetate arms below the nitrogen plane. ${ }^{13}$

The 12-membered, tetraaza-macrocycle of DOTA ${ }^{4-}$ is not large enough to accommodate the actinium ion in the encircled geometry, resulting in the loss of coordination by two acetate groups, and a non-favorable binding energy of $+86 \mathrm{kcal} / \mathrm{mol}$ compared to the traditional encapsulated geometry. Based on this result, the smaller NOTA ${ }^{3-}$ chelator was not tested in the encircled geometry. PEPA ${ }^{5-}$ and $\mathrm{HEHA}^{6-}$ have binding energies with $\mathrm{Ac}^{3+}$ in the encircled geometry of +14.9 and $+13.2 \mathrm{kcal} / \mathrm{mol}$, respectively. $\mathrm{PEPA}^{5-}$ has the potential to coordinate to $\mathrm{Ac}^{3+}$ through ten atoms, but one of the acetate groups lost coordination to the ion in the encircled geometry (see Figure S2), resulting in a final coordination number of nine. Similarly, HEHA ${ }^{6-}$ has a $\mathrm{CN}$ of ten with $\mathrm{Ac}^{3+}$, despite having twelve donor atoms. We did not explore any macrocyclic chelators larger than $\mathrm{HEHA}^{6-}$.

While the binding energies of larger macrocycles ( $\mathrm{PEPA}^{5-}$ and $\mathrm{HEHA}^{6-}$ ) with $\mathrm{Ac}^{3+}$ are similar for either the traditional encapsulated or alternative encircled geometries, there is no encircled geometry that binds $\mathrm{Ac}^{3+}$ more favorably than DOTA ${ }^{4-}$ in the traditional encapsulated configuration $(\mathrm{CN}=8)$. Poorer binding in the encircled geometry could be accounted for by the greater distance the pendant functional groups have to reach the actinium compared to the encapsulated configuration. To test this, we increased the methylene pendant functional groups from one to two carbon atoms as was done in the previous section on DOTA ${ }^{4-}$ to optimize the chelate ring size. However, even with longer functional group arms, the encircled geometries 
were still not favorable $(+89 \mathrm{kcal} / \mathrm{mol}$ for $5 \mathrm{~N}$-macrocycle and $+58 \mathrm{kcal} / \mathrm{mol}$ for $6 \mathrm{~N}-$ macrocycle, from Eqn. (1)).
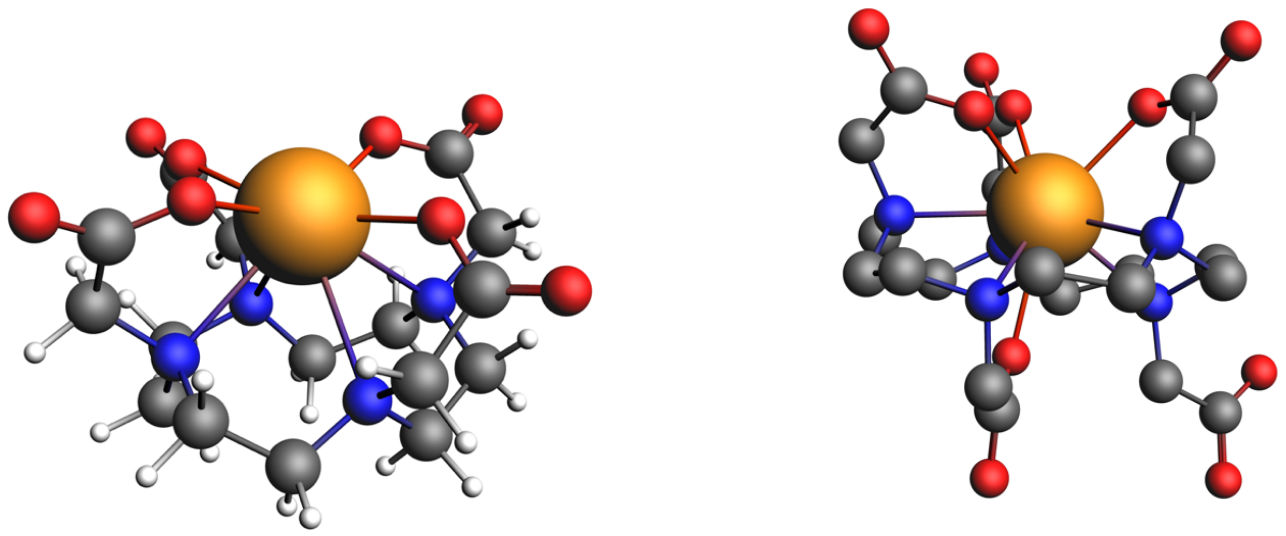

Figure S2: Left: Ac(DOTA) ${ }^{4-}$ in the standard "encapsulated" geometry. Right: Ac(PEPA) ${ }^{2-}$ in the "encircled" configuration with three acetate arms above the $\mathrm{Ac}^{3+}$ ion and two acetate arms below the ion. Note that one acetate group below the $\mathrm{Ac}^{3+}$ has lost coordination to the metal. 


\section{S4. Alternative $\operatorname{Ac}(\text { HEHA })^{3-}$ configurations}

$\mathrm{HEHA}^{6-}$ has six acetate functional groups and is able to coordinate to metals in various configurations based on whether the acetate groups are above or below the nitrogen macrocycle. For $\mathrm{Ac}^{3+}$, three coordination structures were computationally determined to maintain a coordination number of ten (no structures with $\mathrm{CN}>10$ were found). The lowest energy structure had four acetate groups above the macrocycle and two groups below the macrocycle, though the two groups below the ring did not coordinate to $\mathrm{Ac}^{3+}$. All three structures and their energies are shown in Figure S3 and Table S3.
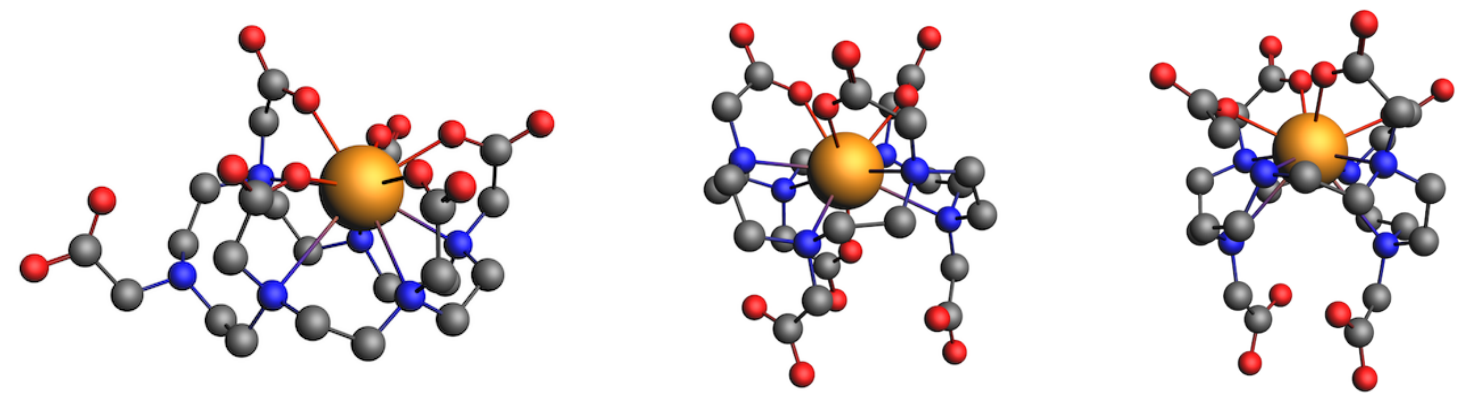

Figure S3: Optimized structures of $A c(H E H A)^{3-}$ with $C N=10$. From left to right: $H E H A$-cage, $H E H A$-ring, HEHA-boat.

\begin{tabular}{|l|l|l|l|}
\hline Structure name & $\mathrm{COO}^{-}$positions & $\mathrm{CN}$ & $\Delta \mathrm{E}$ \\
\hline HEHA-cage & $\mathrm{u}, \mathrm{u}, \mathrm{u}, \mathrm{u}, \mathrm{u}, \mathrm{u}$ & 10 & +27.2 \\
\hline HEHA-ring & $\mathrm{u}, \mathrm{d}, \mathrm{u}, \mathrm{d}, \mathrm{u}, \mathrm{d}$ & 10 & +13.3 \\
\hline HEHA-boat & $\mathrm{u}, \mathrm{u}, \mathrm{d}, \mathrm{u}, \mathrm{u}, \mathrm{d}$ & 10 & +10.6 \\
\hline
\end{tabular}

Table S3: Coordination structures and energies (kcal/mol) from Eqn. (1) of Ac(HEHA) ${ }^{3-}$ with CN=10 with acetate functional groups either up above the macrocycle (u) or down below the macrocycle (d). 


\section{S5. Definition of atomic planes}

For all Ac-chelator complexes, the atomic planes of the nitrogen atoms in the macrocycle, and planes in coordinating oxygen atoms form functional groups are defined in Figure S4.

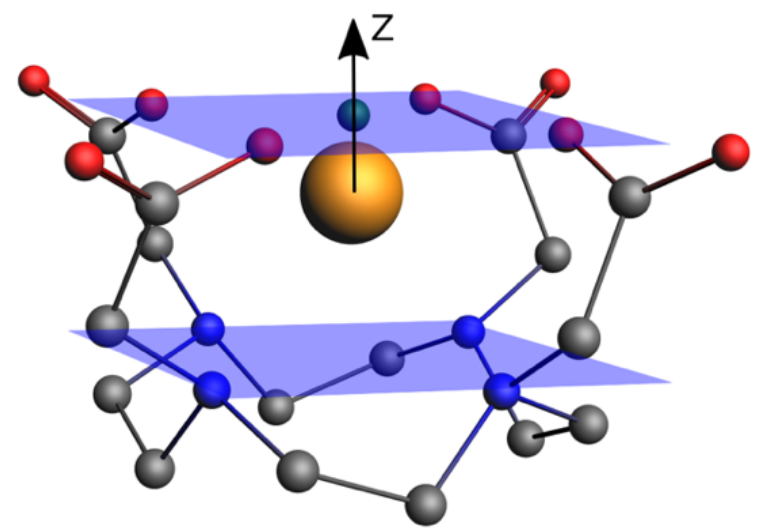

Figure S4: Depiction of oxygen and nitrogen planes in Ac(DOTA)- using the central point of the coordinating oxygen atoms (green sphere) and the metal ion to define the z-axis that is orthogonal to both planes. The two planes were then defined using the average z-coordinates of the oxygen and nitrogen atoms, respectively. Hydrogen atoms are omitted for clarity. 


\section{S6. QTAIM analysis of free ligands and $\mathrm{Ac}^{3+}$ complexes}

Table S4 shows the average values of charge density $(\rho)$ at bond critical points $(\mathrm{CPs})$ for the $\mathrm{Ac}^{3+}$ ion with coordinating oxygen atoms, and with both coordinating and non-coordinating nitrogen atoms. Linear correlations between the amount of charge density at Ac-O and Ac-N bond CPs and the corresponding interatomic distances are plotted in Figure S5. The Ac-O bond CPs have a nearly linear relationship with their distances $\left(R^{2}=0.979\right)$, but the Ac-N bond CP $\rho$ value is lower than expected from the linear trend. When the Ac(HEHP) ${ }^{9-} \mathrm{Ac}-\mathrm{O}$ data point is removed from the fit, the $\mathrm{R}^{2}$ value increases from 0.956 to 0.993 .

\begin{tabular}{|l|l|l|l|l|l|l|}
\hline Bond CP $\boldsymbol{\rho}\left(\mathbf{e} / \AA^{3}\right)$ & DOTA $^{4-}$ & DOTP $^{8-}$ & PEPA $^{5-}$ & PEPP $^{10-}$ & HEHA $^{6-}$ & HEHP $^{12-}$ \\
\hline Ac-O & 0.049 & 0.054 & 0.042 & 0.043 & 0.046 & 0.052 \\
\hline Ac-N (coord.) & 0.033 & 0.028 & 0.022 & 0.016 & 0.031 & 0.025 \\
\hline Ac-N (non-coord.) & & & & & 0.012 & 0.005 \\
\hline
\end{tabular}

Table S4: Average value of charge density ( $\rho$ ) at bond critical points (CPs) between Ac and O/N atoms for Ac ${ }^{3+}$ complexes. For HEHA(P) complexes, properties concerning nitrogen atoms are separated into coordinating (coord.) and non-coordinating (noncoord.) atoms.
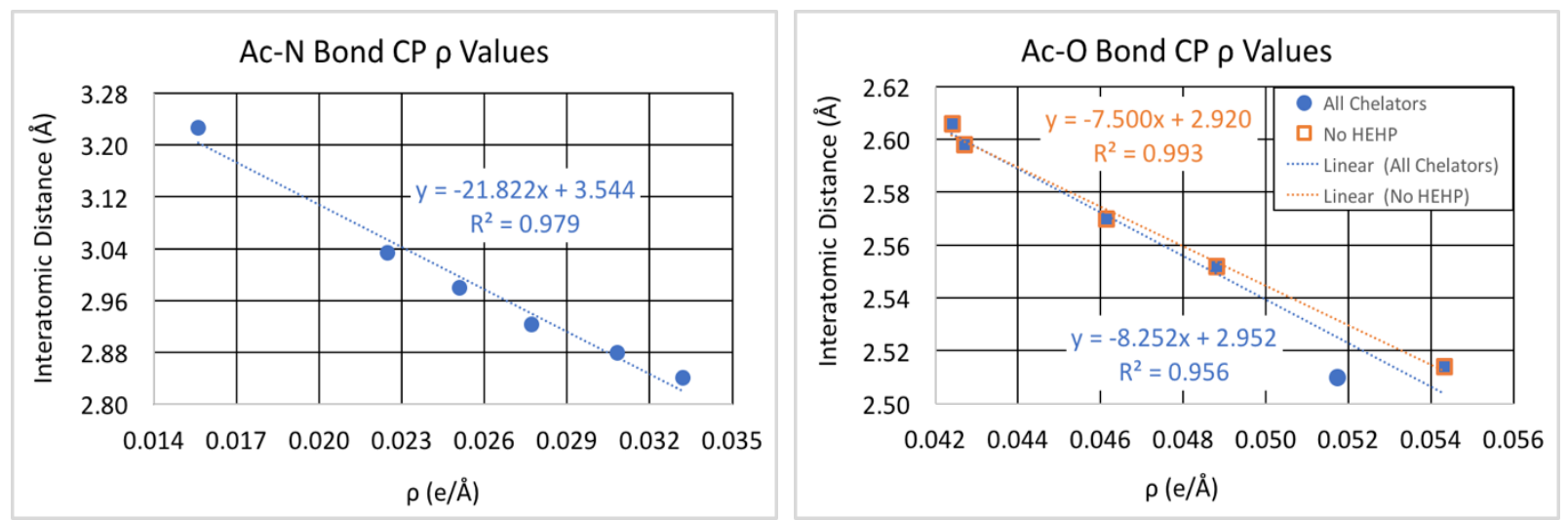

Figure S5: Linear relationships between bond distances and values of $\rho$ at bond CPs for Ac-N (left) and Ac-O (right). Only coordinated nitrogen atoms are included.

Table S5 shows the $\mathrm{Ac}^{3+}$-ligand complex and the free ligand Bader atomic charges. The average charge transfer from metal complexation for coordinating oxygen atoms on the acetate and phosphonate functional groups is 0.067 electrons. There is low charge transfer $(<0.1$ electrons) for all atoms coordinating to $\mathrm{Ac}^{3+}$, indicative of ionic interactions. For the nitrogen atoms in the macrocycle, the charge transfer is even smaller, 0.012 for the coordinating nitrogen atoms and 0.008 for non-coordinating nitrogen atoms in $\mathrm{HEHA}^{6-}$ and $\mathrm{HEHP}^{12-}$. 


\begin{tabular}{|c|c|c|c|c|c|c|}
\hline Bader Charge (e) & & & & & & \\
\hline Complex & DOTA $^{4-}$ & DOTP $^{8-}$ & PEPA $^{5-}$ & $\mathrm{PEPP}^{10-}$ & HEHA $^{6-}$ & HEHP $^{12-}$ \\
\hline Ac & +2.345 & +2.369 & +2.383 & +2.405 & +2.355 & +2.378 \\
\hline $\mathrm{O}$ & -1.304 & -1.596 & -1.323 & -1.595 & -1.311 & -1.581 \\
\hline $\mathrm{N}$ (coord.) & -1.015 & -1.034 & -1.010 & -1.008 & -1.030 & -1.011 \\
\hline $\mathrm{N}$ (non-coord.) & & & & & -1.021 & -1.008 \\
\hline Free Ligand & DOTA $^{4-}$ & DOTP $^{8-}$ & PEPA $^{5-}$ & $\mathrm{PEPP}^{10-}$ & HEHA $^{6-}$ & HEHP $^{12-}$ \\
\hline $\mathrm{O}$ & -1.369 & -1.674 & -1.363 & -1.671 & -1.358 & -1.670 \\
\hline $\mathrm{N}$ & -1.018 & -1.012 & -1.020 & -1.022 & -1.018 & -1.022 \\
\hline$\Delta$ Charge & DOTA $^{4-}$ & DOTP $^{8-}$ & PEPA $^{5-}$ & PEPP $^{10-}$ & HEHA $^{6-}$ & HEHP $^{12-}$ \\
\hline $\mathrm{O}$ & 0.065 & 0.078 & 0.040 & 0.076 & 0.047 & 0.089 \\
\hline $\mathrm{N}$ (coord.) & 0.003 & -0.022 & 0.010 & 0.014 & -0.012 & 0.011 \\
\hline $\mathrm{N}$ (non-coord.) & & & & & -0.003 & 0.014 \\
\hline
\end{tabular}

Table S5: Bader atomic charges for Ac ${ }^{3+}$ complexes and free ligands, and the change in Bader charges after metal complexation. 


\section{S7. Mulliken Charges}

Mulliken charges for $\mathrm{Ac}^{3+}$ complexes are shown in Table S6. While Mulliken charges are calculated using a vastly different method than Bader charges, the same qualitative trends on all atoms hold for the complexes studied here.

\begin{tabular}{|l|r|r|r|r|r|r|}
\hline Mulliken Charge (e) & \multicolumn{1}{|c|}{ DOTA $^{4-}$} & \multicolumn{1}{|c|}{ DOTP $^{8-}$} & PEPA $^{5-}$ & PEPP $^{10-}$ & \multicolumn{1}{|c|}{ HEHA $^{6-}$} & HEHP $^{2-}$ \\
\hline Ac & 1.953 & 1.196 & 1.988 & 2.081 & 2.027 & 2.157 \\
\hline O & -0.857 & -0.997 & -0.804 & -0.980 & -0.796 & -0.950 \\
\hline N (coord) & -0.427 & -0.441 & -0.342 & -0.325 & -0.358 & -0.319 \\
\hline N (non-coord) & & & & & -0.348 & -0.325 \\
\hline
\end{tabular}

Table S6: Mulliken Charges for Ac ${ }^{3+}$ complexes. 


\section{S8. Energy Decomposition Analysis (EDA) Results}

Table $\mathrm{S} 7$ shows the results from EDA, which separates the bonding energy in to three categories: Pauli repulsion, electrostatic, and orbital relaxation (details in S1a). The orbital relaxation energy is considered the covalent part of the bonding, while the electrostatic interactions are the ionic component.

\begin{tabular}{|l|r|r|r|r|r|r|}
\hline & \multicolumn{1}{|c|}{ DOTA $^{4-}$} & \multicolumn{1}{|c|}{ DOTP $^{8-}$} & PEPA $^{5-}$ & PEPP $^{10-}$ & HEHA $^{6-}$ & HEHP $^{12-}$ \\
\hline Pauli (P) & 202.9 & 201.8 & 168.3 & 151.6 & 182.2 & 188.1 \\
\hline Electrostatic (E) & -1215.6 & -1950.3 & -1380.8 & -2199.0 & -1494.2 & -2452.1 \\
\hline Steric (E+P) & -1012.8 & -1748.6 & -1212.4 & -2047.3 & -1312.0 & -2264.0 \\
\hline Orbital & -332.8 & -336.65 & -330.4 & -315.2 & -362.7 & -359.8 \\
\hline \% Ionic & 79 & 85 & 81 & 87 & 80 & 87 \\
\hline \% Covalent & 21 & 15 & 19 & 13 & 20 & 13 \\
\hline
\end{tabular}

Table S7: Bonding energy decomposition results for chelators complexed with Ac, energies in kcal/mol. 


\section{S9. Natural Orbitals of Chemical Valence (NOCV) Results}

Table S8 and S9 show NOCVs for $\mathrm{Ac}^{3+}$-chelator complexes with acetate groups and

phosphonate groups, respectively. Only NOCVs that involve contributions from both the chelator and metal, or that have an eigenvalue $(v)>0.3$ are included. Eigenvalues and energy contributions $(\Delta \mathrm{E})$ in $\mathrm{kcal} / \mathrm{mol}$ are listed with an image of each NOCV.

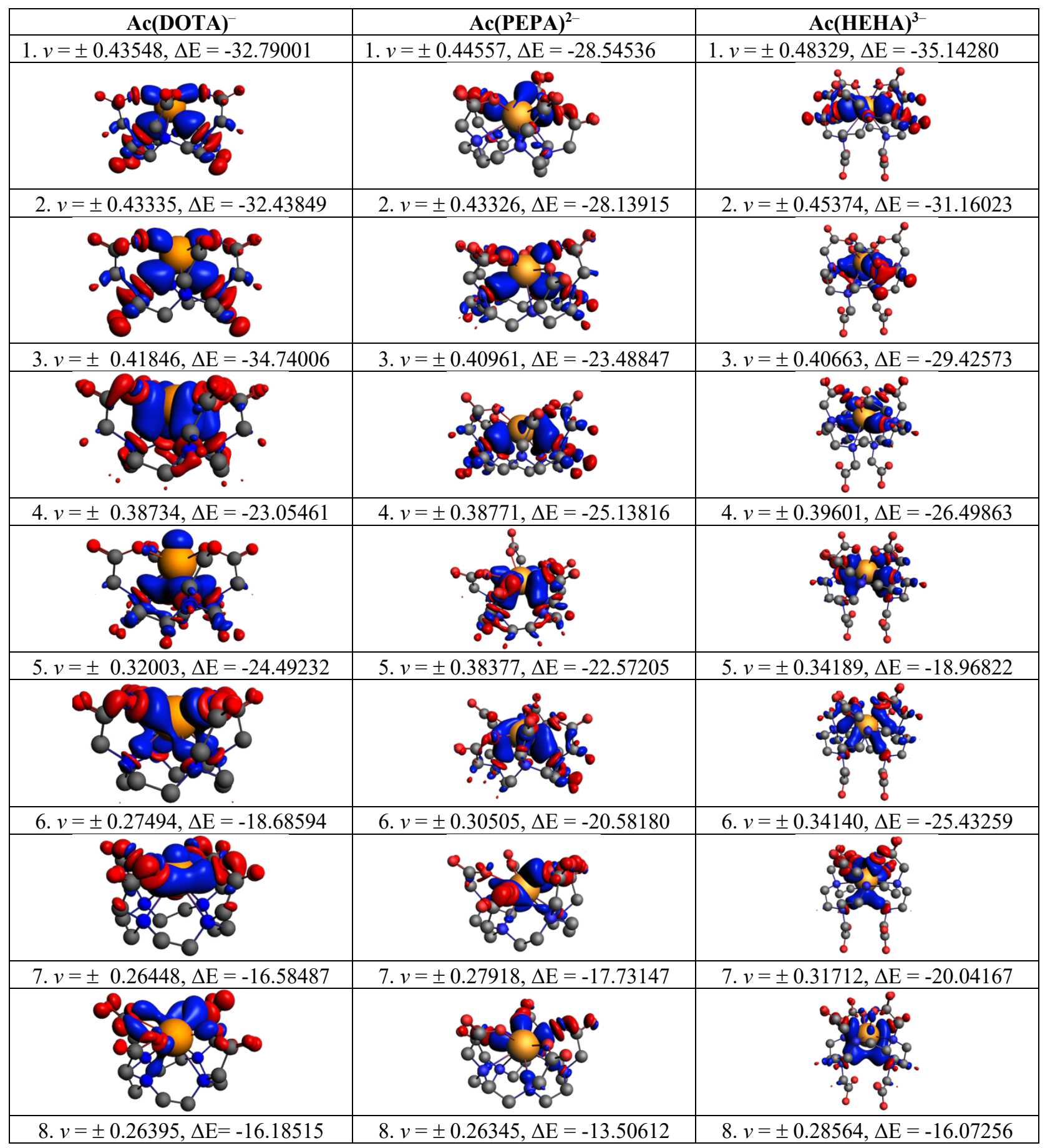




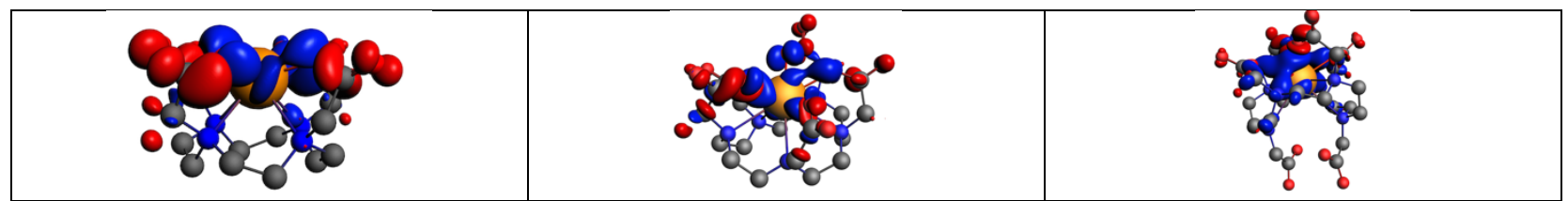

Table S8: Images of NOCVs for Ac(DOTA)-, Ac(PEPA) ${ }^{2-}$, and Ac(HEHA) ${ }^{3-}$ using an isosurface value of 0.0005 e. Listed above each image is the eigenvalue (v) and energy contribution in kcal/mol to the covalent bonding between the Ac ${ }^{3+}$ and ligand.

\begin{tabular}{|c|c|c|}
\hline Ac(DOTP) $)^{5-}$ & Ac(PEPP $)^{7-}$ & Ac(HEHP) $)^{9-}$ \\
\hline 1. $v= \pm 0.76928, \Delta \mathrm{E}=-36.39897$ & 1. $v= \pm 1.42538, \Delta \mathrm{E}=-62.90053$ & 1. $v= \pm 0.62118, \Delta \mathrm{E}=-35.40200$ \\
\hline 2. $v= \pm 0.57855, \Delta \mathrm{E}=-33.76414$ & 2. $v= \pm 0.66343, \Delta \mathrm{E}=-27.43165$ & 2. $v= \pm 0.60255, \Delta \mathrm{E}=-36.32808$ \\
\hline 3. $v= \pm 0.57553, \Delta \mathrm{E}=-32.83845$ & 3. $v= \pm 0.59566, \Delta \mathrm{E}=-24.89411$ & 3. $v= \pm 0.58154, \Delta \mathrm{E}=-30.27412$ \\
\hline 4. $v= \pm 0.49896, \Delta \mathrm{E}=-27.74933$ & 4. $v= \pm 0.49092, \Delta \mathrm{E}=-18.39963$ & 4. $v= \pm 0.57097, \Delta \mathrm{E}=-29.46811$ \\
\hline $5 . v= \pm 0.39087, \Delta \mathrm{E}=-36.64751$ & $5 . v= \pm 0.45701, \Delta \mathrm{E}=-13.94144$ & $5 . v= \pm 0.40745, \Delta \mathrm{E}=-34.45917$ \\
\hline 6. $v= \pm 0.32191, \Delta \mathrm{E}=-19.04356$ & 6. $v= \pm 0.35758, \Delta \mathrm{E}=-30.29953$ & 6. $v= \pm 0.36975, \Delta \mathrm{E}=-24.22647$ \\
\hline 7. $v= \pm 0.31658, \Delta \mathrm{E}=-18.38538$ & 7. $v= \pm 0.34106, \Delta \mathrm{E}=-26.33068$ & 7. $v= \pm 0.32529, \Delta \mathrm{E}=-13.91697$ \\
\hline & & \\
\hline
\end{tabular}




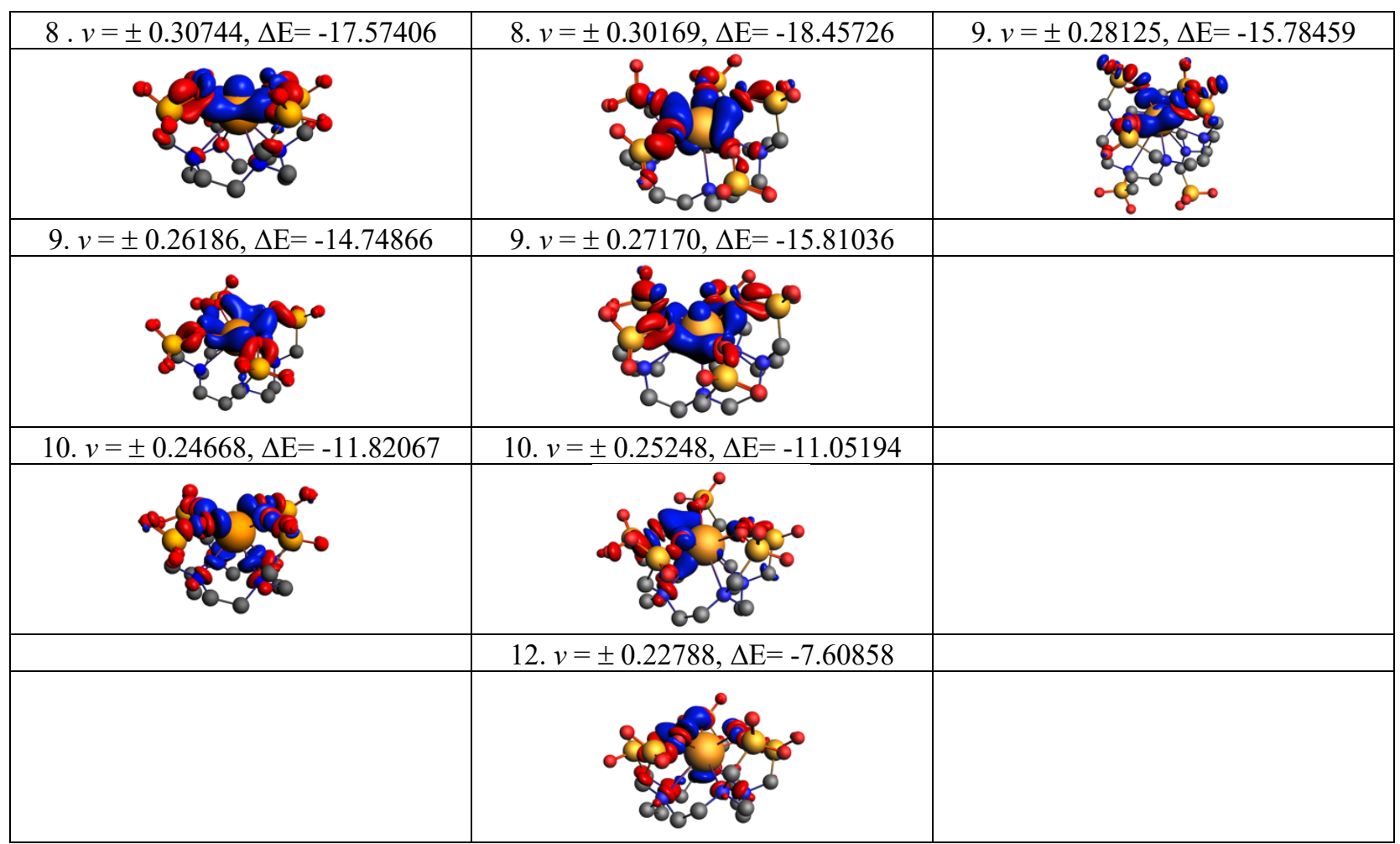

Table S9: Images of NOCVs for Ac(DOTP $)^{5-}, A c(P E P P)^{7-}$, and $A c(H E H P)^{9-}$ using an isosurface value of 0.0005 e. Listed above each image is the eigenvalue (v) and energy contribution in $\mathrm{kcal} / \mathrm{mol}$ to the covalent bonding between the $\mathrm{Ac}^{3+}$ and ligand. 


\section{S10. $4 \mathrm{~N}$-macrocycles with three $\mathrm{PO}_{3}{ }^{2-}$ functional groups}

Table S10 shows the energies for $4 \mathrm{~N}$-macrocyles with three $\mathrm{PO}_{3}{ }^{2-}$ groups and an alternative functional group $\left(\mathrm{OCH}_{3}, \mathrm{NCH}_{3}\right.$, or $\left.\mathrm{CH}_{3}\right)$ either coordinating or non-coordinating to the $\mathrm{Ac}^{3+}$. All complexes with a non-coordinating functional group have their lowest energy when one phosphonate group has bidentate coordination to $\mathrm{Ac}^{3+}$ as shown in Figure S6. This results in a coordination number of eight for all geometries.

\begin{tabular}{|l|l|l|}
\hline Functional Group & Coord. $\Delta \mathrm{G}$ & Non-coord. $\Delta \mathrm{G}$ \\
\hline $\mathrm{OCH}_{3}$ & -13.18 & -13.24 \\
\hline $\mathrm{NCH}_{3}$ & -18.36 & -15.71 \\
\hline $\mathrm{CH}_{3}$ & -13.07 & -15.44 \\
\hline
\end{tabular}

Table S10: Energies (kcal/mol) for $4 \mathrm{~N}$-macrocyles with three $\mathrm{PO}_{3}{ }^{2-}$ functional groups plus one alternative functional group that is either coordinated (coord.) or non-coordinated (non-coord.) to $\mathrm{Ac}^{3+}$.
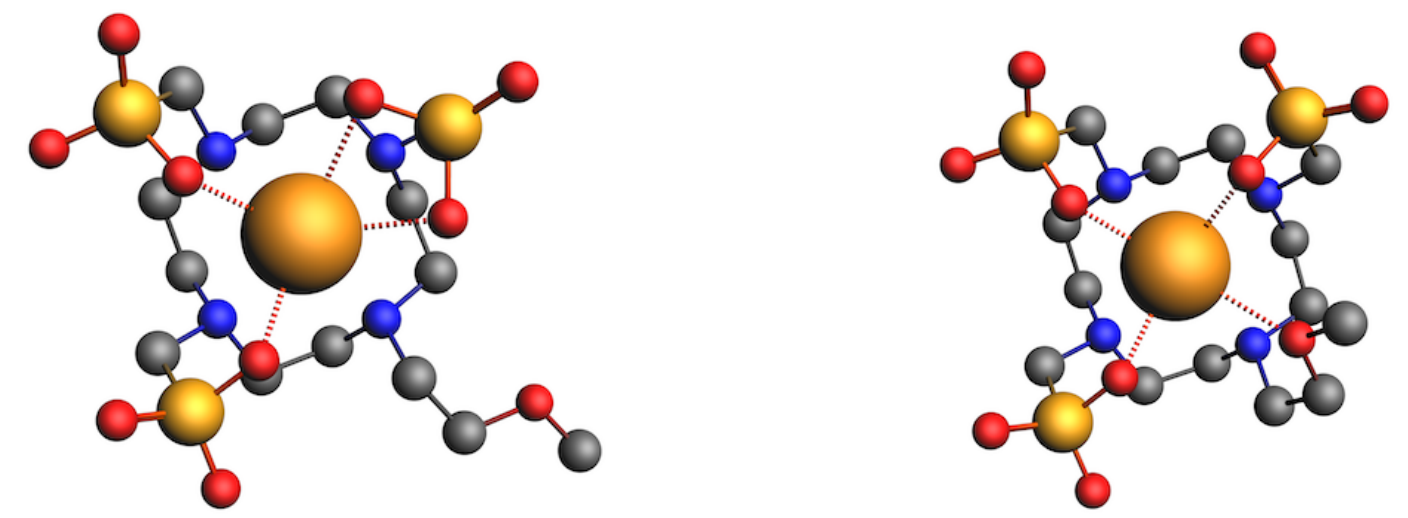

Figure S6: Top-down view of $\mathrm{Ac}^{3+}$ coordinated to $4 \mathrm{~N}$-marcrocycles with three $\mathrm{PO}_{3}{ }^{2-}$ groups and $1 \mathrm{OCH}_{3}$ group. Left: Noncoordinated $\mathrm{OCH}_{3}$, resulting in bidentate phosphonate bonding. Right: Coordinated $\mathrm{OCH}_{3}$ group. All hydrogen atoms are omitted for clarity. 


\section{S11. Ac-Complex Coordinates}

Optimized coordinates for Ac-complexes using PBE0/TZ2P with small frozen core, scalar ZORA, and COSMO solvation with water $(\varepsilon=78.39)$

\begin{tabular}{|c|c|c|c|}
\hline \multicolumn{4}{|c|}{$\operatorname{Ac}(\mathrm{DOTA})^{-}$} \\
\hline Ac & 0.02364096 & -0.28297112 & -0.14066427 \\
\hline $\mathrm{C}$ & 0.43128584 & 0.94260915 & -3.33212239 \\
\hline $\mathrm{C}$ & -0.30654916 & -2.06296423 & -3.27407110 \\
\hline $\mathrm{O}$ & 0.49647377 & 1.77312261 & -4.26615446 \\
\hline $\mathrm{C}$ & 3.32708036 & -1.06624741 & 0.83641451 \\
\hline $\mathrm{H}$ & 4.35386252 & -1.46716753 & 0.76820690 \\
\hline $\mathrm{H}$ & 3.02732000 & -1.13091966 & 1.89351957 \\
\hline $\mathrm{N}$ & 2.38399977 & -1.85902924 & 0.01510783 \\
\hline $\mathrm{C}$ & 2.13158296 & -3.18293051 & 0.64415132 \\
\hline $\mathrm{H}$ & 3.07515246 & -3.62326688 & 1.01556831 \\
\hline $\mathrm{H}$ & 1.76440498 & -3.86432613 & -0.13432523 \\
\hline $\mathrm{C}$ & 1.13715995 & -3.12214442 & 1.79795269 \\
\hline $\mathrm{H}$ & 1.07817817 & -4.12138357 & 2.26827555 \\
\hline $\mathrm{H}$ & 1.50554499 & -2.43436046 & 2.57167459 \\
\hline $\mathrm{O}$ & 4.35966528 & 1.09461462 & 0.79292906 \\
\hline $\mathrm{O}$ & -2.48951811 & 0.05127351 & 0.16520497 \\
\hline $\mathrm{H}$ & -0.93218812 & -1.24712158 & -3.66136453 \\
\hline $\mathrm{H}$ & 0.02528313 & -2.64831914 & -4.15150235 \\
\hline $\mathrm{C}$ & 1.33815341 & -0.29972330 & -3.40990074 \\
\hline $\mathrm{H}$ & 1.47096504 & -0.58925359 & -4.46714218 \\
\hline $\mathrm{H}$ & 2.32376243 & 0.02029632 & -3.03900735 \\
\hline $\mathrm{N}$ & 0.86276343 & -1.44003597 & -2.59290117 \\
\hline $\mathrm{C}$ & 1.95299143 & -2.43446399 & -2.40618315 \\
\hline $\mathrm{H}$ & 2.48952952 & -2.60674630 & -3.35756497 \\
\hline $\mathrm{H}$ & 1.49555677 & -3.39520910 & 00626 \\
\hline $\mathrm{C}$ & 2.96667018 & -2.02306292 & -1.34583022 \\
\hline $\mathrm{H}$ & 3.78434237 & -2.76758654 & -1.32771062 \\
\hline $\mathrm{H}$ & 3.42537436 & -1.06383104 & -1.62225429 \\
\hline $\mathrm{O}$ & -0.31048539 & 1.06313850 & -2.28016733 \\
\hline $\mathrm{O}$ & 2.27915421 & 0.91578839 & -0.07012212 \\
\hline $\mathrm{C}$ & 3.34257472 & 0.4 & 154 \\
\hline $\mathrm{H}$ & -0.51547726 & -3.78029363 & -1.97613390 \\
\hline $\mathrm{C}$ & -0.96083149 & -2.25422542 & 2.61898542 \\
\hline $\mathrm{H}$ & -0.89446087 & -3.02899509 & 3.40320783 \\
\hline $\mathrm{H}$ & -2.02699076 & -2.14216729 & 2.37077254 \\
\hline $\mathrm{N}$ & -0.22046822 & -2.65535638 & 1.40063150 \\
\hline $\mathrm{C}$ & -0.95533849 & -3.72634707 & 0.67616966 \\
\hline $\mathrm{H}$ & -1.33208836 & -4.4 & 7445 \\
\hline $\mathrm{H}$ & -0.24345465 & -4.24537216 & 0.02163961 \\
\hline $\mathrm{C}$ & -2.12871439 & -3.20031919 & -0.14170166 \\
\hline $\mathrm{H}$ & -2.67464004 & -4.05941941 & -0.57370446 \\
\hline $\mathrm{H}$ & -2.83813842 & -2.68057359 & 0.51670287 \\
\hline $\mathrm{O}$ & 0.07724433 & -0.07912931 & 2.40068905 \\
\hline $\mathrm{H}$ & -1.92406851 & -3.44636293 & -2.97746257 \\
\hline $\mathrm{O}$ & -0.79260730 & -0.6 & 9773 \\
\hline $\mathrm{C}$ & -0.51646587 & -0.90026286 & 3.20365262 \\
\hline $\mathrm{C}$ & -2.93796863 & -1.49262378 & -1.64111418 \\
\hline $\mathrm{H}$ & -3.78076000 & -2.17288443 & -1.85651219 \\
\hline $\mathrm{H}$ & -2.71293156 & -0.95479053 & -2.57473004 \\
\hline $\mathrm{N}$ & -1.73529355 & -2.24357800 & -1.21360598 \\
\hline $\mathrm{C}$ & -1.13688906 & -2.96509409 & -2.36852095 \\
\hline $\mathrm{C}$ & -3.39164227 & -0.42303761 & -0.62999773 \\
\hline $\mathrm{O}$ & -4.58675136 & -0.05270380 & -0.66723693 \\
\hline & (ETA) ${ }^{-}$ & & \\
\hline $\mathrm{C}$ & -1.68827700 & 2.8 & 800 \\
\hline $\mathrm{C}$ & 0.54923700 & 2.66167200 & 2.36774100 \\
\hline $\mathrm{N}$ & 1.40879000 & 2.04491000 & 1.32321600 \\
\hline $\mathrm{C}$ & 2.69508900 & 1.61562900 & 1.92892600 \\
\hline $\mathrm{C}$ & 2.86336900 & -0.98053800 & 1.80965400 \\
\hline
\end{tabular}




\begin{tabular}{|c|c|c|c|}
\hline $\mathrm{N}$ & 1.65176900 & -1.77786100 & 1.47751700 \\
\hline $\mathrm{C}$ & 0.97995200 & -2.24726300 & 2.71652500 \\
\hline $\mathrm{C}$ & -1.62393600 & -1.81191500 & 2.67666700 \\
\hline $\mathrm{N}$ & -1.95143200 & -1.64815800 & 1.23827600 \\
\hline $\mathrm{C}$ & -3.41732700 & -1.40943100 & 1.06992900 \\
\hline $\mathrm{C}$ & -3.38144800 & 1.15524900 & 0.86954300 \\
\hline $\mathrm{N}$ & -2.22647500 & 1.76793300 & 0.17876400 \\
\hline $\mathrm{C}$ & 1.74418200 & 3.03336100 & 0.28181500 \\
\hline $\mathrm{C}$ & 2.40880900 & 2.40923400 & -0.95198700 \\
\hline $\mathrm{O}$ & 2.19892000 & 1.16792100 & -1.14193000 \\
\hline $\mathrm{O}$ & 3.07745900 & 3.14983400 & -1.68980900 \\
\hline $\mathrm{O}$ & 3.09812900 & -3.35230700 & -1.45132900 \\
\hline $\mathrm{C}$ & 2.31590400 & -2.63219100 & -0.81371400 \\
\hline $\mathrm{C}$ & 2.12019800 & -2.92350000 & 0.67846300 \\
\hline $\mathrm{O}$ & 1.60192500 & -1.70130100 & -1.31024200 \\
\hline $\mathrm{O}$ & -2.08380900 & -3.71619300 & -1.68747600 \\
\hline $\mathrm{C}$ & -1.73642400 & -2.72364700 & -1.03365200 \\
\hline $\mathrm{C}$ & -1.59691200 & -2.86556900 & 0.49242400 \\
\hline $\mathrm{O}$ & -1.43826300 & -1.58251700 & -1.52374700 \\
\hline $\mathrm{O}$ & -1.77022900 & 3.19112600 & -3.12664700 \\
\hline $\mathrm{C}$ & -1.57900700 & 2.42472500 & -2.17224000 \\
\hline $\mathrm{C}$ & -2.67483100 & 2.30412900 & -1.11212200 \\
\hline $\mathrm{O}$ & -0.55360600 & 1.67797600 & -2.02474300 \\
\hline $\mathrm{H}$ & -1.02919300 & 3.46870400 & 0.35783500 \\
\hline $\mathrm{H}$ & -2.51366500 & 3.54782000 & 1.27741000 \\
\hline $\mathrm{H}$ & 0.86886900 & 2.26718200 & 3.33716400 \\
\hline $\mathrm{H}$ & 0.73765300 & 3.74412500 & 2.40362200 \\
\hline $\mathrm{H}$ & 3.03629900 & 2.41122200 & 2.60857400 \\
\hline $\mathrm{H}$ & 3.43790700 & 1.55594400 & 1.12827200 \\
\hline $\mathrm{H}$ & 3.55945200 & -1.65676700 & 2.32932300 \\
\hline $\mathrm{H}$ & 3.34550400 & -0.70875100 & 0.86258700 \\
\hline $\mathrm{H}$ & 1.71379800 & -2.27231600 & 3.53324200 \\
\hline $\mathrm{H}$ & 0.66052600 & -3.28442400 & 2.57690900 \\
\hline $\mathrm{H}$ & -1.83002800 & -2.84711600 & 2.99360200 \\
\hline $\mathrm{H}$ & -2.31778200 & -1.17805600 & 3.23450600 \\
\hline $\mathrm{H}$ & -3.87307600 & -1.32987900 & 2.06213400 \\
\hline $\mathrm{H}$ & -3.87148200 & -2.29422700 & 0.60575900 \\
\hline $\mathrm{H}$ & -4.21720400 & 1.87277700 & 0.86249900 \\
\hline $\mathrm{H}$ & -3.117 & 00 & 41600 \\
\hline $\mathrm{H}$ & 2.39445900 & 3.82340300 & 0.68450900 \\
\hline $\mathrm{H}$ & 0.83757600 & 3.53155000 & -0.07373800 \\
\hline $\mathrm{H}$ & -3.17503000 & 3.27515600 & -0.98835200 \\
\hline $\mathrm{H}$ & 3.04868000 & -3.34534200 & 1.08748500 \\
\hline $\mathrm{H}$ & 1.37759500 & -3.72802600 & 0.71801000 \\
\hline $\mathrm{H}$ & -3.41912500 & 1.62767400 & -1.54635600 \\
\hline $\mathrm{H}$ & -0.54859500 & -3.12019300 & 0.68266400 \\
\hline $\mathrm{H}$ & -2.19228700 & -3.72669700 & 0.82694700 \\
\hline $\mathrm{Ac}$ & 0.09358200 & 0.00443200 & -0.29730200 \\
\hline $\mathrm{C}$ & 2.70390300 & 0.28309200 & 2.67382500 \\
\hline $\mathrm{C}$ & -0.22622800 & -1.40685700 & 3.14545500 \\
\hline $\mathrm{C}$ & -3.81489300 & -0.18169200 & 0.25844200 \\
\hline $\mathrm{C}$ & -0.95078200 & 2.39048200 & 2.25737700 \\
\hline $\mathrm{H}$ & -1.12664200 & 1.31334000 & 2.37941000 \\
\hline $\mathrm{H}$ & -1.40934700 & 2.84160200 & 3.14319400 \\
\hline $\mathrm{H}$ & 1.86234500 & 0.20402100 & 3.36831000 \\
\hline $\mathrm{H}$ & 3.58810000 & 0.31759100 & 3.31926200 \\
\hline $\mathrm{H}$ & -0.05106300 & -0.35175400 & 2.90162900 \\
\hline $\mathrm{H}$ & -0.27610500 & -1.43278100 & 4.24052500 \\
\hline $\mathrm{H}$ & -4.90744000 & -0.20385000 & 0.17800300 \\
\hline $\mathrm{H}$ & -3.43410500 & -0.27634300 & -0.76312600 \\
\hline
\end{tabular}

\section{Ac(DOTPA)-}

$\begin{array}{lccc}\text { Ac } & -0.13592200 & -0.06344300 & 0.07215700 \\ \text { C } & 1.07924800 & 2.15023400 & -2.44369000 \\ \text { C } & -0.59868200 & -1.98217300 & -3.11672300 \\ \text { O } & 1.48940600 & 3.31744100 & -2.54486300 \\ \text { C } & 3.92391000 & 0.36074900 & 0.50400800 \\ \text { H } & 4.26810100 & 0.52214900 & -0.52372500 \\ \text { H } & 4.82138100 & 0.49677100 & 1.11788400\end{array}$




\begin{tabular}{|c|c|c|c|}
\hline & 2.34171500 & -1.58258000 & -0.11613100 \\
\hline c & 2.05338600 & -2.96485600 & 0.32077000 \\
\hline & 2.99636900 & 3.52714100 & 0.41956900 \\
\hline & 48818400 & 4500 & 578600 \\
\hline & 1.29838300 & -3.07881700 & 3948400 \\
\hline $\mathrm{H}$ & 1.30145800 & -4.13769000 & 359400 \\
\hline$H$ & 1.83690400 & 32800 & 4300 \\
\hline & .43174300 & 65321300 & 7500 \\
\hline c & -2.45334000 & .88650300 & 330600 \\
\hline & -1.36546100 & -1.34284100 & 4200 \\
\hline & -0.26293700 & -2.64757300 & 3.92782500 \\
\hline & 1.62 & & 0900 \\
\hline $\mathrm{H}$ & & & \\
\hline $\mathrm{H}$ & 2.6 & & \\
\hline $\mathrm{r}$ & 0.5 & & \\
\hline c & & & \\
\hline & 2.25 & & 7500 \\
\hline & 1.42 & & \\
\hline C & 2.7 & & \\
\hline $\mathrm{H}$ & & & \\
\hline & & & \\
\hline & 0.2 & & \\
\hline c & 1.7 & & 100 \\
\hline & & & \\
\hline H & -0.4 & & 2700 \\
\hline c & & & \\
\hline H & & & \\
\hline & & & \\
\hline & -0 . & & 600 \\
\hline & -0.9 & -3. & 000 \\
\hline & & & \\
\hline $\mathrm{H}$ & -0.3 & & 000 \\
\hline c & -2.17 & & \\
\hline & & & \\
\hline & -2 & & \\
\hline & -0.8 & & 600 \\
\hline & -1. & & 200 \\
\hline c & & & \\
\hline C & -0.3 & & \\
\hline C & & & \\
\hline & & & 300 \\
\hline & & & \\
\hline $\mathrm{r}$ & -1.9 & -2.0 & 36800 \\
\hline c & -1.2 & & 91200 \\
\hline C & -3.3 & & \\
\hline $\mathrm{O}$ & -4.2 & & 2500 \\
\hline C & & & \\
\hline $\mathrm{H}$ & & & \\
\hline & & & \\
\hline C & 0.68 & -0.0 & 500 \\
\hline & -0.3 & & 8700 \\
\hline $\mathrm{H}$ & & & \\
\hline 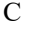 & -3.2. & -1.57801300 & -1.46157400 \\
\hline $\mathrm{H}$ & & & 400 \\
\hline & & & 1400 \\
\hline & & & \\
\hline & & & \\
\hline & -1.69548600 & -2.19593000 & 2.866649 \\
\hline
\end{tabular}

$\begin{array}{lccc}\text { Ac(NOTA) } & & \\ \text { Ac } & -0.15491700 & -1.16852100 & 0.13618700 \\ \text { H } & -2.95017200 & -0.78367900 & -3.33490500 \\ \text { H } & -2.87124300 & -1.58689000 & -1.76672100 \\ \text { O } & 0.31691300 & -3.55843900 & 0.36345300 \\ \text { C } & 2.73409200 & 0.04212800 & -1.41606500 \\ \text { H } & 3.29549900 & 0.71372900 & -2.07876300 \\ \text { H } & 3.31571400 & -0.88337700 & -1.33027500 \\ \text { N } & 1.41084600 & -0.28046800 & -1.97378900 \\ \text { C } & 1.53570500 & -1.26225900 & -3.08014500\end{array}$




$\begin{array}{crrr}\mathrm{H} & 2.58019800 & -1.34543900 & -3.39858200 \\ \mathrm{H} & 0.99617700 & -0.89643300 & -3.95516700 \\ \mathrm{C} & 1.05312000 & -2.65443300 & -2.69284300 \\ \mathrm{H} & 1.15846500 & -3.31906400 & -3.56350400 \\ \mathrm{H} & 1.70324700 & -3.05898200 & -1.90634800 \\ \mathrm{O} & 3.66614400 & 1.29632300 & 0.38448000 \\ \mathrm{O} & -2.42974700 & -0.42849300 & 0.63866200 \\ \mathrm{C} & -3.13427300 & 0.42844100 & -0.01112000 \\ \mathrm{O} & 0.10131600 & -5.70564400 & -0.21534100 \\ \mathrm{C} & -0.01803200 & -4.50118900 & -0.44406800 \\ \mathrm{C} & -2.53555400 & 0.93462600 & -1.32903500 \\ \mathrm{H} & -3.34942500 & 1.17292400 & -2.02621700 \\ \mathrm{H} & -2.03514300 & 1.87927800 & -1.08567100 \\ \mathrm{C} & -0.69074300 & 0.75246400 & -2.90190400 \\ \mathrm{H} & -1.11759700 & 1.73561300 & -3.12661000 \\ \mathrm{H} & -0.67646600 & 0.22058700 & -3.85451000 \\ \mathrm{C} & 0.72568800 & 0.96550300 & -2.38281700 \\ \mathrm{H} & 1.30794400 & 1.49282500 & -3.15339100 \\ \mathrm{H} & 0.69606000 & 1.62286400 & -1.50441200 \\ \mathrm{~N} & -1.55461400 & 0.02314300 & -1.93988000 \\ \mathrm{O} & 1.65495100 & 0.37508900 & 0.69743500 \\ \mathrm{C} & 2.69714700 & 0.64372000 & -0.00583600 \\ \mathrm{O} & -4.20940200 & 0.89480600 & 0.36830400 \\ \mathrm{C} & -0.66885900 & -4.06209800 & -1.76151200 \\ \mathrm{H} & -0.42096200 & -4.78920100 & -2.54581200 \\ \mathrm{H} & -1.75073800 & -4.12570800 & -1.59519800 \\ \mathrm{~N} & -0.33277200 & -2.69008100 & -2.17521900 \\ \mathrm{C} & -1.32905300 & -2.17692800 & -3.14883000 \\ \mathrm{H} & -1.94967400 & -2.99699400 & -3.52500300 \\ \mathrm{H} & -0.81233700 & -1.78186800 & -4.02499900 \\ \mathrm{C} & -2.25740400 & -1.12639100 & -2.55157100\end{array}$

\section{Ac(PEPA) $)^{2-}$}

$\mathrm{N} \quad-3.99782000$

C $\quad-3.81939600$

C $\quad-3.21021800$

$\mathrm{N} \quad-1.81346700$

C $\quad-0.84871100$

C -0.38123000

$\mathrm{N} \quad 0.35606600$

C $\quad 0.71612900$

C -0.39978600

$\mathrm{N} \quad-0.75796600$

C $\quad-1.87086400$

C -2.59054400

$\mathrm{N} \quad-3.28105300$

C $\quad-4.50116400$

C $\quad-4.34388400$

O $\quad-2.14147200$

C $\quad-2.04919700$

C $\quad-1.60097800$

$\mathrm{H} \quad-3.57900500$

$\mathrm{H}-0.52824300$

H $\quad-2.06947700$

O $\quad-2.24421400$

O 2.38797800

$-0.46540200$

0.77242300

1.92098200

1.74256400

2.16026600

1.08009900

$-0.02765600$

$-0.99716800$

$-1.95044400$

$-2.89148600$

$-3.73647700$

$-4.41811500$

$-3.50966700$

$-2.93662000$

$-1.56398600$

0.59357100

1.85336100

2.52921000

$-1.59197700$

2.69436200

3.52248100

2.60007000

1.49270200

0.78748900

0.45572500

$-1.33527600$

$-0.33560800$

1.32014100

0.29418300

$-3.85983500$

$-3.29824400$

$-3.74957900$

$-3.61345800$

$-4.73248200$

$\begin{array}{lllr}\mathrm{H} & 1.01772400 & -3.93248300 & -1.22170100 \\ \mathrm{O} & 0.81964700 & -2.43539800 & 1.62134100\end{array}$

0.59590900

$-0.18904400$

0.60291400

1.04040200

0.00684100

$-0.95291400$

$-0.32492800$

$-1.37594800$

$-1.75561900$

$-0.68553900$

$-1.14713900$

$-0.00141100$

0.92478300

0.32629000

$-0.32477000$

3.56929500

3.56094900

2.26153800

$-1.10587000$

2.39959300

2.19432700

4.54475900

2.30682700

1.83479400

0.34056800

$-0.83425300$

0.30050900

$-0.18485800$

2.49926300

0.91605400

0.82188800

$-0.34074600$

$-0.44174600$

$-0.03381500$

$\begin{array}{lllr}\mathrm{H} & 1.01772400 & -3.93248300 & -1.22170100 \\ \mathrm{O} & 0.81964700 & -2.43539800 & 1.62134100\end{array}$ 


$\begin{array}{lccc}\mathrm{O} & -2.45891400 & -5.22924600 & 4.01274500 \\ \mathrm{C} & -2.41204400 & -4.32693900 & 3.15264700 \\ \mathrm{C} & -3.57064000 & -4.26846600 & 2.14611600 \\ \mathrm{H} & -5.27265000 & -2.87285400 & 1.09818900 \\ \mathrm{H} & -3.88868300 & -5.29703200 & 1.91923800 \\ \mathrm{H} & -4.38777800 & -3.78984200 & 2.68982400 \\ \mathrm{O} & -1.50653600 & -3.44053000 & 3.07802200 \\ \mathrm{O} & -5.88933000 & -1.14415200 & 3.64442400 \\ \mathrm{C} & -4.88611300 & -1.07425200 & 2.89875900 \\ \mathrm{C} & -5.05952000 & -0.28621400 & 1.59659500 \\ \mathrm{Ac} & -1.46905700 & -1.21012700 & 1.81216900 \\ \mathrm{H} & -6.04532400 & -0.51110800 & 1.16379500 \\ \mathrm{H} & -5.09955900 & 0.76202200 & 1.90398900 \\ \mathrm{O} & -3.75248400 & -1.56427700 & 3.13951700 \\ \mathrm{H} & -4.78744900 & 1.11164700 & -0.59307900 \\ \mathrm{H} & -3.19812800 & 0.53630500 & -1.05816700 \\ \mathrm{H} & -3.81435800 & 2.09348100 & 1.49776800 \\ \mathrm{H} & -3.30395400 & 2.83692300 & -0.00223500 \\ \mathrm{H} & 0.03171100 & 2.57092200 & 0.50850900 \\ \mathrm{H} & -1.25983600 & 2.98565000 & -0.59539600 \\ \mathrm{H} & 0.26155500 & 1.57559200 & -1.70232600 \\ \mathrm{H} & -1.22560100 & 0.66372800 & -1.50868100 \\ \mathrm{H} & 1.57605800 & -1.57739100 & -1.03341800 \\ \mathrm{H} & 1.04806800 & -0.47210100 & -2.28640600 \\ \mathrm{H} & -1.30068200 & -1.39447100 & -2.04061200 \\ \mathrm{H} & -0.07964800 & -2.50010900 & -2.65809400 \\ \mathrm{H} & -1.50761300 & -4.50942500 & -1.84417600 \\ \mathrm{H} & -2.56674300 & -3.12297500 & -1.72406000 \\ \mathrm{H} & -1.86677800 & -4.99374100 & 0.58462500 \\ \mathrm{H} & -3.30329100 & -5.14999100 & -0.41814400\end{array}$

$\begin{array}{lrrc}\text { Ac }(\mathrm{HEHA})^{3-} & & \\ \mathrm{N} & -3.48461200 & -0.33606700 & -1.06903000 \\ \mathrm{C} & -3.76260900 & 1.01887200 & -0.56367100 \\ \mathrm{C} & -3.19246700 & 1.35546700 & 0.80324500 \\ \mathrm{~N} & -1.73061600 & 1.20078600 & 0.95724300 \\ \mathrm{C} & -0.99689500 & 1.94407600 & -0.08198000 \\ \mathrm{C} & 0.51497900 & 1.77658500 & -0.01819500 \\ \mathrm{~N} & 0.19506100 & -2.04854000 & -2.48762100 \\ \mathrm{C} & 0.60104600 & -3.45959400 & -2.59548100 \\ \mathrm{C} & 0.87719500 & -4.17801000 & -1.28629000 \\ \mathrm{~N} & -0.22516200 & -4.18551600 & -0.30171800 \\ \mathrm{C} & -1.47295400 & -4.69526000 & -0.89624000 \\ \mathrm{C} & -2.66512500 & -4.68010800 & 0.05035200 \\ \mathrm{~N} & -3.12967100 & -3.33053000 & 0.43853500 \\ \mathrm{C} & -4.00203000 & -2.77693900 & -0.61914800 \\ \mathrm{C} & -4.39717500 & -1.30776700 & -0.43249100 \\ \mathrm{O} & 0.08414100 & -0.22294400 & 2.58040600 \\ \mathrm{C} & -0.23001100 & 0.92880000 & 3.00589500 \\ \mathrm{C} & -1.38048200 & 1.65856400 & 2.30701700 \\ \mathrm{H} & -5.41189800 & -1.17291500 & -0.84454300 \\ \mathrm{H} & -1.18578500 & 2.74109700 & 2.32493200 \\ \mathrm{H} & -2.24261900 & 1.48712600 & 2.96108600 \\ \mathrm{O} & 0.28800500 & 1.49737200 & 3.98788800 \\ \mathrm{O} & -1.74618100 & -2.24949400 & -5.59283500 \\ \mathrm{C} & -1.57164900 & -2.41205000 & -4.35614500 \\ \mathrm{C} & -0.36615100 & -1.64074500 & -3.78624300 \\ \mathrm{H} & -4.46878100 & -1.06815200 & 0.62898200 \\ \mathrm{H} & -0.68094300 & -0.59460400 & -3.72601100 \\ \mathrm{H} & 0.42365400 & -1.69250100 & -4.55578100 \\ \mathrm{O} & -2.29476800 & -3.10421900 & -3.60048200 \\ \mathrm{O} & -0.24572500 & -5.43942600 & 3.12612500 \\ \mathrm{C} & -0.30221300 & -4.58524600 & 2.21874100 \\ \mathrm{C} & 0.21108300 & -5.00060200 & 0.83795200 \\ \mathrm{H} & -3.49188600 & -2.90048800 & -1.57975100 \\ \mathrm{H} & -0.00733200 & -6.06650800 & 0.67553400 \\ \mathrm{H} & 1.30019400 & -4.91405800 & 0.92176400 \\ \mathrm{O} & -0.69471400 & -3.38923800 & 2.36665400 \\ \mathrm{O} & -4.43901900 & -2.11042400 & 3.62680600 \\ & & & \\ & & \end{array}$




$\begin{array}{cccc}\mathrm{C} & -3.68344300 & -2.16400100 & 2.63496900 \\ \mathrm{C} & -3.83702700 & -3.38659000 & 1.72369500 \\ \mathrm{H} & -4.92381200 & -3.38036600 & -0.66576900 \\ \mathrm{H} & -3.43761300 & -4.21700700 & 2.31386400 \\ \mathrm{H} & -4.90906900 & -3.59242300 & 1.58576700 \\ \mathrm{O} & -2.78768600 & -1.31688900 & 2.34942800 \\ \mathrm{O} & -3.60770000 & 0.80065500 & -4.55502000 \\ \mathrm{C} & -3.04881300 & 0.63384100 & -3.43862400 \\ \mathrm{C} & -3.78419800 & -0.35051900 & -2.51064000 \\ \mathrm{Ac} & -0.89019600 & -1.53622600 & 0.61407600 \\ \mathrm{H} & -4.86234200 & -0.16636200 & -2.65979600 \\ \mathrm{H} & -3.57554900 & -1.34806100 & -2.90864700 \\ \mathrm{O} & -1.97738700 & 1.17087100 & -3.06934400 \\ \mathrm{H} & -4.85297600 & 1.18945400 & -0.50919800 \\ \mathrm{H} & -3.37589900 & 1.72650500 & -1.29749200 \\ \mathrm{H} & -3.65105000 & 0.73362400 & 1.57679500 \\ \mathrm{H} & -3.48168700 & 2.40039800 & 1.01150600 \\ \mathrm{H} & -1.21348600 & 3.02415800 & 0.00092000 \\ \mathrm{H} & -1.35886200 & 1.61718700 & -1.06169200 \\ \mathrm{H} & 0.87264500 & 2.04648300 & 0.97888500 \\ \mathrm{H} & 0.96443400 & 2.50730100 & -0.71001000 \\ \mathrm{H} & 1.51477300 & -3.54761800 & -3.21072300 \\ \mathrm{H} & -0.18612300 & -3.98639000 & -3.13532000 \\ \mathrm{H} & 1.14792500 & -5.21497400 & -1.55163200 \\ \mathrm{H} & 1.74056300 & -3.73893900 & -0.77908500 \\ \mathrm{H} & -1.33264900 & -5.73744300 & -1.23474100 \\ \mathrm{H} & -1.70305700 & -4.09649700 & -1.78310300 \\ \mathrm{H} & -2.41706300 & -5.22929300 & 0.96234400 \\ \mathrm{H} & -3.48351000 & -5.24159500 & -0.42874600 \\ \mathrm{C} & 1.38248700 & -1.20263900 & -2.24824600 \\ \mathrm{C} & 1.07328700 & 0.22767400 & -1.79279800 \\ \mathrm{~N} & 1.00125800 & 0.41417500 & -0.32780800 \\ \mathrm{H} & 1.97697300 & -1.14486700 & -3.17612500 \\ \mathrm{H} & 2.01748700 & -1.70143000 & -1.51528100 \\ \mathrm{H} & 0.12179000 & 0.55280600 & -2.22530500 \\ \mathrm{H} & 1.85410200 & 0.89503300 & -2.19399100 \\ \mathrm{C} & 2.31315000 & 0.18769700 & 0.29203200 \\ \mathrm{C} & 2.59502800 & -1.23758100 & 0.78174800 \\ \mathrm{H} & 2.39305200 & 0.80463500 & 1.19234600 \\ \mathrm{H} & 3.13259600 & 0.50162600 & -0.37144100 \\ \mathrm{O} & 1.62496500 & -2.04744400 & 0.84813300 \\ \mathrm{O} & 3.77009400 & -1.48855900 & 1.11815800\end{array}$

$\begin{array}{lccc}\mathrm{Ac}(\mathrm{DOTP}) & & & \\ \mathrm{Ac} & 0.02094657 & -0.17466930 & -0.04379932 \\ \mathrm{O} & 4.62728766 & 0.72510711 & -0.34930827 \\ \mathrm{O} & 2.14964770 & 1.13440374 & 0.25342233 \\ \mathrm{O} & 3.97629032 & 1.23111892 & 2.10369178 \\ \mathrm{C} & 3.23579316 & -1.19251351 & 1.11894226 \\ \mathrm{H} & 4.20789442 & -1.71604345 & 1.23143884 \\ \mathrm{H} & 2.73009330 & -1.20690023 & 2.09519216 \\ \mathrm{~N} & 2.36767373 & -1.89473472 & 0.13950249 \\ \mathrm{C} & 2.04517317 & -3.25362180 & 0.63681622 \\ \mathrm{H} & 2.96589598 & -3.75684833 & 0.99552368 \\ \mathrm{H} & 1.68163264 & -3.84978118 & -0.21109805 \\ \mathrm{C} & 1.01751846 & -3.26734913 & 1.76394547 \\ \mathrm{H} & 0.90792386 & -4.30997341 & 2.12549857 \\ \mathrm{H} & 1.37238392 & -2.67379020 & 2.61766718 \\ \mathrm{P} & 3.54921951 & 0.61016215 & 0.75443558 \\ \mathrm{O} & -0.75356149 & 0.93918713 & -4.46777920 \\ \mathrm{O} & -0.23617555 & 1.40601306 & -1.97583630 \\ \mathrm{O} & 1.23151022 & 2.47529941 & -3.83192180 \\ \mathrm{C} & 1.51981930 & -0.17411557 & -3.30554734 \\ \mathrm{H} & 1.84622608 & -0.46921769 & -4.32472472 \\ \mathrm{H} & 2.40254814 & 0.20781093 & -2.77261039 \\ \mathrm{~N} & 1.00084043 & -1.34645379 & -2.55572322 \\ \mathrm{C} & 2.09043802 & -2.32914709 & -2.32986312 \\ \mathrm{H} & 2.69533757 & -2.44300834 & -3.25185210 \\ \mathrm{H} & 1.63044525 & -3.30945989 & -2.14572531\end{array}$




$\begin{array}{lccc}\mathrm{C} & 3.03148641 & -1.96710982 & -1.18506056 \\ \mathrm{H} & 3.85721388 & -2.70839038 & -1.16589168 \\ \mathrm{H} & 3.49791915 & -0.98812006 & -1.36537563 \\ \mathrm{P} & 0.34536330 & 1.27170971 & -3.43224956 \\ \mathrm{O} & 0.83747763 & -1.35521653 & 4.35133736 \\ \mathrm{O} & -0.04087713 & 0.16029577 & 2.44459631 \\ \mathrm{O} & -1.58274101 & -0.43323170 & 4.44864742 \\ \mathrm{C} & -1.08212670 & -2.34005369 & 2.57801422 \\ \mathrm{H} & -1.20158835 & -3.21322025 & 3.25296704 \\ \mathrm{H} & -2.08752497 & -2.03449926 & 2.25261217 \\ \mathrm{~N} & -0.30210517 & -2.71471564 & 1.36903596 \\ \mathrm{C} & -1.04792656 & -3.71287770 & 0.56047836 \\ \mathrm{H} & -1.49561775 & -4.48066843 & 1.22284047 \\ \mathrm{H} & -0.32211941 & -4.24353250 & -0.07067001 \\ \mathrm{C} & -2.15776444 & -3.12341535 & -0.30508904 \\ \mathrm{H} & -2.70153801 & -3.96230301 & -0.78719514 \\ \mathrm{H} & -2.89339967 & -2.59281546 & 0.31586438 \\ \mathrm{P} & -0.40478157 & -0.89223712 & 3.55793607 \\ \mathrm{O} & -4.59463342 & -1.03301899 & 0.25036538 \\ \mathrm{O} & -2.42730689 & 0.37306664 & 0.18617102 \\ \mathrm{O} & -4.34667053 & 0.84946818 & -1.50772487 \\ \mathrm{C} & -2.81060631 & -1.36505882 & -1.86440867 \\ \mathrm{H} & -3.58038627 & -2.02769112 & -2.31227738 \\ \mathrm{H} & -2.42331612 & -0.71502548 & -2.66213004 \\ \mathrm{~N} & -1.67725376 & -2.16234311 & -1.32839636 \\ \mathrm{C} & -0.99245651 & -2.87819034 & -2.43333421 \\ \mathrm{H} & -1.73804086 & -3.37487030 & -3.08691387 \\ \mathrm{H} & -0.38638500 & -3.68263023 & -1.99472990 \\ \mathrm{C} & -0.11539499 & -1.98050066 & -3.30048842 \\ \mathrm{H} & 0.27465014 & -2.58412166 & -4.14546384 \\ \mathrm{H} & -0.71057402 & -1.16955590 & -3.74274032 \\ \mathrm{P} & -3.63255263 & -0.21323957 & -0.64176681\end{array}$

$\begin{array}{cccc}\text { Ac(PEPP) } & 7 & & \\ \mathrm{~N} & -3.90958400 & -0.24363400 & 0.60103800 \\ \mathrm{C} & -3.58783000 & 0.90136500 & -0.27257200 \\ \mathrm{C} & -2.88506000 & 2.06449700 & 0.41372300 \\ \mathrm{~N} & -1.51672900 & 1.80265300 & 0.86828800 \\ \mathrm{C} & -0.51876500 & 2.06280700 & -0.18011800 \\ \mathrm{C} & -0.19916000 & 0.93392900 & -1.14318100 \\ \mathrm{~N} & 0.42003500 & -0.24336000 & -0.53952100 \\ \mathrm{C} & 0.59376400 & -1.25855200 & -1.59234900 \\ \mathrm{C} & -0.62277400 & -2.11492400 & -1.88894400 \\ \mathrm{~N} & -0.97777500 & -3.03770200 & -0.81559900 \\ \mathrm{C} & -2.19198500 & -3.76622600 & -1.18616300 \\ \mathrm{C} & -2.89998000 & -4.36727400 & 0.01231800 \\ \mathrm{~N} & -3.46376000 & -3.39236400 & 0.94527500 \\ \mathrm{C} & -4.62378200 & -2.68973900 & 0.37238400 \\ \mathrm{C} & -4.36279300 & -1.33370800 & -0.27680200 \\ \mathrm{O} & -1.90547300 & 0.70161400 & 3.70824900 \\ \mathrm{P} & -1.99633600 & 2.23516400 & 3.61526900 \\ \mathrm{C} & -1.18647200 & 2.65780200 & 2.02255900 \\ \mathrm{H} & -3.61748300 & -1.42901900 & -1.07145800 \\ \mathrm{H} & -0.11685700 & 2.54300300 & 2.20879800 \\ \mathrm{H} & -1.37918500 & 3.72390100 & 1.79122300 \\ \mathrm{O} & -3.42841800 & 2.76301300 & 3.57904700 \\ \mathrm{O} & 2.06453300 & 2.18882900 & 1.74627600 \\ \mathrm{P} & 1.93983100 & 0.65975300 & 1.77162900 \\ \mathrm{C} & 1.74858300 & 0.03160500 & 0.04166100 \\ \mathrm{H} & -5.30595400 & -1.03379300 & -0.77487300 \\ \mathrm{H} & 2.25151000 & -0.93619000 & 0.11108100 \\ \mathrm{H} & 2.34260700 & 0.67515900 & -0.63690500 \\ \mathrm{O} & 0.70520200 & 0.17969500 & 2.54928100 \\ \mathrm{O} & 2.62037200 & -3.10196100 & 0.05263800 \\ \mathrm{P} & 1.36297600 & -3.61576000 & 0.76420300 \\ \mathrm{C} & 0.08635100 & -4.01295200 & -0.52389400 \\ \mathrm{H} & -5.10407600 & -3.32042700 & -0.39796200 \\ \mathrm{H} & -0.39171100 & -4.90500600 & -0.11339200 \\ \mathrm{H} & 0.58592800 & -4.32593900 & -1.46022300 \\ & & & \\ & & & \end{array}$




$\begin{array}{lccc}\mathrm{O} & 0.69785700 & -2.57939600 & 1.66825400 \\ \mathrm{O} & -1.73835200 & -5.73867900 & 2.77028300 \\ \mathrm{P} & -2.48847700 & -4.53139500 & 3.32883500 \\ \mathrm{C} & -3.85212500 & -4.10137400 & 2.17524900 \\ \mathrm{H} & -5.35983000 & -2.54824600 & 1.16733700 \\ \mathrm{H} & -4.41086100 & -5.02797000 & 1.93760800 \\ \mathrm{H} & -4.50389200 & -3.44320900 & 2.74642200 \\ \mathrm{O} & -1.59210800 & -3.27597800 & 3.43670900 \\ \mathrm{O} & -6.27233800 & -1.87730700 & 2.88951200 \\ \mathrm{P} & -5.18139900 & -0.81793600 & 3.11186300 \\ \mathrm{C} & -4.94549300 & 0.17543600 & 1.56851100 \\ \mathrm{Ac} & -1.42717900 & -1.17519700 & 2.02652800 \\ \mathrm{H} & -5.90784400 & 0.33146000 & 1.04645200 \\ \mathrm{H} & -4.63358700 & 1.13411800 & 1.98922300 \\ \mathrm{O} & -3.79736100 & -1.41233200 & 3.35646300 \\ \mathrm{H} & -4.51728500 & 1.28901300 & -0.73064900 \\ \mathrm{H} & -2.97444100 & 0.53074200 & -1.09878900 \\ \mathrm{H} & -3.46197300 & 2.37678900 & 1.28796300 \\ \mathrm{H} & -2.89621900 & 2.91846300 & -0.28915200 \\ \mathrm{H} & 0.41420800 & 2.33263700 & 0.32167700 \\ \mathrm{H} & -0.82633100 & 2.93909300 & -0.78127500 \\ \mathrm{H} & 0.48211700 & 1.36111200 & -1.90896400 \\ \mathrm{H} & -1.09239000 & 0.61132900 & -1.68722200 \\ \mathrm{H} & 1.40753600 & -1.92113600 & -1.28950300 \\ \mathrm{H} & 0.91001800 & -0.77316500 & -2.53534100 \\ \mathrm{H} & -1.49778400 & -1.48724300 & -2.10133400 \\ \mathrm{H} & -0.40861500 & -2.67260400 & -2.82425400 \\ \mathrm{H} & -1.95663700 & -4.57923800 & -1.89919600 \\ \mathrm{H} & -2.86606200 & -3.09098800 & -1.72066200 \\ \mathrm{H} & -2.20648700 & -4.99200100 & 0.58370300 \\ \mathrm{H} & -3.70150300 & -5.03712400 & -0.35859100 \\ \mathrm{O} & -1.15448500 & 2.93213800 & 4.69310900 \\ \mathrm{O} & -5.64167100 & 0.19064300 & 4.16557200 \\ \mathrm{O} & -3.18508700 & -4.85165700 & 4.65793100 \\ \mathrm{O} & 1.64721500 & -4.95029500 & 1.45932300 \\ \mathrm{O} & 3.24414600 & 0.02180600 & 2.26215400\end{array}$

$\begin{array}{lccc}\text { Ac(HEHP) } & & & \\ \mathrm{N} & -3.57669300 & -0.30736500 & -1.21955200 \\ \mathrm{C} & -3.85863800 & 0.98892600 & -0.61791200 \\ \mathrm{C} & -3.25780200 & 1.24998000 & 0.75423600 \\ \mathrm{~N} & -1.78439500 & 1.19481800 & 0.89000400 \\ \mathrm{C} & -1.13312800 & 1.92166900 & -0.20368900 \\ \mathrm{C} & 0.38242400 & 1.81633000 & -0.20151700 \\ \mathrm{~N} & 0.15415700 & -2.04581200 & -2.70674900 \\ \mathrm{C} & 0.64861200 & -3.41622400 & -2.75933200 \\ \mathrm{C} & 1.00573900 & -4.05312200 & -1.42748600 \\ \mathrm{~N} & -0.06236600 & -4.16112500 & -0.40869600 \\ \mathrm{C} & -1.30435000 & -4.66663000 & -1.00029600 \\ \mathrm{C} & -2.48751700 & -4.67963100 & -0.04685200 \\ \mathrm{~N} & -2.98403900 & -3.35008800 & 0.36912900 \\ \mathrm{C} & -3.81237200 & -2.79011200 & -0.71742700 \\ \mathrm{C} & -4.30277900 & -1.35455200 & -0.49842200 \\ \mathrm{O} & 0.31965600 & -0.15848500 & 2.75025100 \\ \mathrm{P} & 0.01005400 & 1.28515900 & 3.18913200 \\ \mathrm{C} & -1.50279500 & 1.75606800 & 2.22839700 \\ \mathrm{H} & -5.37236200 & -1.30891700 & -0.78781100 \\ \mathrm{H} & -1.59163900 & 2.85834400 & 2.20498400 \\ \mathrm{H} & -2.30078900 & 1.38678800 & 2.87758100 \\ \mathrm{O} & -0.43653200 & 1.37026300 & 4.65033100 \\ \mathrm{O} & -2.09723800 & -1.98206400 & -6.05593700 \\ \mathrm{P} & -1.55716800 & -2.84606600 & -4.91219900 \\ \mathrm{C} & -0.50341700 & -1.65253300 & -3.96367700 \\ \mathrm{H} & -4.28799100 & -1.12654800 & 0.56690100 \\ \mathrm{H} & -1.13368800 & -0.77350600 & -3.78483300 \\ \mathrm{H} & 0.26845700 & -1.34202800 & -4.69804500 \\ \mathrm{O} & -2.63955000 & -3.38535200 & -3.97798500 \\ \mathrm{O} & -1.20287500 & -5.88411600 & 2.66282300 \\ \mathrm{P} & -0.05322100 & -4.91195300 & 2.40626600\end{array}$




$\begin{array}{cccc}\mathrm{C} & 0.49823100 & -5.03513000 & 0.64257000 \\ \mathrm{H} & -3.24415000 & -2.84659500 & -1.65276000 \\ \mathrm{H} & 0.45605100 & -6.09311200 & 0.32210400 \\ \mathrm{H} & 1.55524900 & -4.76839700 & 0.71867200 \\ \mathrm{O} & -0.46745000 & -3.44045300 & 2.59002800 \\ \mathrm{O} & -5.17038000 & -1.35302200 & 2.60592500 \\ \mathrm{P} & -3.87207500 & -2.12681400 & 2.83457300 \\ \mathrm{C} & -3.73943100 & -3.53188000 & 1.62881400 \\ \mathrm{H} & -4.69247200 & -3.44888800 & -0.84473900 \\ \mathrm{H} & -3.18765700 & -4.27440600 & 2.21143100 \\ \mathrm{H} & -4.74253700 & -3.94900700 & 1.42360700 \\ \mathrm{O} & -2.62301500 & -1.27196400 & 2.56189500 \\ \mathrm{O} & -4.15551800 & 0.60330500 & -5.12083000 \\ \mathrm{P} & -3.73759200 & 1.14174100 & -3.74868800 \\ \mathrm{C} & -3.95219800 & -0.33980600 & -2.64595000 \\ \mathrm{Ac} & -0.68021500 & -1.57625300 & 0.96138300 \\ \mathrm{H} & -5.03496100 & -0.56813100 & -2.72909300 \\ \mathrm{H} & -3.42247400 & -1.16560100 & -3.13472600 \\ \mathrm{O} & -2.27790900 & 1.59013300 & -3.67404300 \\ \mathrm{H} & -4.95177500 & 1.14370800 & -0.50266900 \\ \mathrm{H} & -3.53657500 & 1.76018500 & -1.31586200 \\ \mathrm{H} & -3.66034300 & 0.55793600 & 1.49960900 \\ \mathrm{H} & -3.60118500 & 2.26544700 & 1.03144100 \\ \mathrm{H} & -1.39002400 & 2.99846700 & -0.15389000 \\ \mathrm{H} & -1.53053400 & 1.54596100 & -1.15140400 \\ \mathrm{H} & 0.75672500 & 2.11763300 & 0.77954000 \\ \mathrm{H} & 0.77683800 & 2.54475700 & -0.93444400 \\ \mathrm{H} & 1.56243100 & -3.48272300 & -3.38567900 \\ \mathrm{H} & -0.08979900 & -4.02446700 & -3.27775000 \\ \mathrm{H} & 1.36696900 & -5.07043400 & -1.67495500 \\ \mathrm{H} & 1.83235200 & -3.52070100 & -0.94852000 \\ \mathrm{H} & -1.16145300 & -5.70512800 & -1.36110000 \\ \mathrm{H} & -1.54679200 & -4.05844400 & -1.87646500 \\ \mathrm{H} & -2.20783200 & -5.22874000 & 0.85531200 \\ \mathrm{H} & -3.30640500 & -5.24587000 & -0.52923400 \\ \mathrm{O} & -3.85531600 & -2.80002300 & 4.20881800 \\ \mathrm{O} & -4.7310827500 & -2.27548200 & 3.21664400 \\ \mathrm{C} & 0.87921400 & -1.13503200 & -2.43706100 \\ \mathrm{~N} & 0.92070400 & 0.26435100 & -1.95829300 \\ \mathrm{H} & 1.86774600 & -1.02438300 & -0.49987000 \\ \mathrm{H} & 1.93830900 & -1.59585500 & -3.36255700 \\ \mathrm{H} & -0.12953600 & 0.50396000 & -1.71296700 \\ \mathrm{H} & 1.56170400 & 0.99840300 & -2.42748900 \\ \mathrm{C} & 2.29516600 & 0.40473300 & 0.04251700 \\ \mathrm{P} & 3.00818000 & -1.23899400 & 0.51990500 \\ \mathrm{H} & 2.27293500 & 0.92784200 & 1.00226100 \\ \mathrm{H} & 3.00117200 & 0.94486900 & -0.61492700 \\ \mathrm{O} & 1.78354700 & -2.09446900 & 0.87735600 \\ \mathrm{O} & 3.90044600 & -0.93289600 & 1.72544100 \\ \mathrm{O} & 1.15188300 & 2.25170500 & 2.88557200 \\ & -0.65491100 & -1.82647800 & -0.63677700 \\ & & -3.96886600 & -5.45973900 \\ \end{array}$

\section{Ac(PETP-H $)^{5-}$}

$\begin{array}{lrrr}\mathrm{N} & -4.17627400 & -0.76789400 & 0.20398700 \\ \mathrm{C} & -3.96428900 & 0.39345800 & -0.66878200 \\ \mathrm{C} & -3.75009500 & 1.70865400 & 0.07151300 \\ \mathrm{~N} & -2.57037300 & 1.76524500 & 0.95681300 \\ \mathrm{C} & -1.46133000 & 2.49920800 & 0.32954300 \\ \mathrm{C} & -0.70521700 & 1.72709100 & -0.74088600 \\ \mathrm{~N} & 0.03578000 & 0.56150500 & -0.24312800 \\ \mathrm{C} & 0.49443800 & -0.26319700 & -1.37427800 \\ \mathrm{C} & -0.46969200 & -1.36976100 & -1.77227600 \\ \mathrm{~N} & -0.74788500 & -2.24730200 & -0.63492100 \\ \mathrm{C} & -1.33519500 & -3.53227900 & -1.01186900 \\ \mathrm{C} & -1.90143000 & -4.26759900 & 0.19404700 \\ \mathrm{~N} & -2.90057300 & -3.51497800 & 0.96624000\end{array}$




$\begin{array}{cccc}\mathrm{C} & -4.14076400 & -3.27794100 & 0.20530000 \\ \mathrm{C} & -4.18304000 & -1.98835300 & -0.60838500 \\ \mathrm{O} & -1.07720800 & 0.83847900 & 3.44630800 \\ \mathrm{P} & -1.63470100 & 2.27746800 & 3.57188600 \\ \mathrm{C} & -2.92801500 & 2.37239100 & 2.25390200 \\ \mathrm{H} & -3.33603900 & -1.93260600 & -1.29393500 \\ \mathrm{H} & -3.23636900 & 3.42666400 & 2.13274200 \\ \mathrm{H} & -3.78233000 & 1.81820100 & 2.65710100 \\ \mathrm{O} & -2.38184500 & 2.50980700 & 4.87951100 \\ \mathrm{O} & 2.94796200 & -1.14556700 & 0.45668000 \\ \mathrm{P} & 2.02566000 & -0.44448100 & 1.45224800 \\ \mathrm{C} & 1.17172200 & 0.96187700 & 0.60311500 \\ \mathrm{H} & -5.09267400 & -2.02468500 & -1.23597900 \\ \mathrm{H} & 1.91246900 & 1.54910900 & 0.02938800 \\ \mathrm{H} & 0.80235100 & 1.59857600 & 1.41311300 \\ \mathrm{O} & 0.86311100 & -1.36053200 & 1.89654300 \\ \mathrm{H} & -1.08722900 & -4.52312000 & 0.88105000 \\ \mathrm{H} & -2.11373900 & -3.35652200 & -1.76067700 \\ \mathrm{H} & -0.59731900 & -4.18998800 & -1.49862700 \\ \mathrm{H} & -4.33866900 & -4.12311400 & -0.47607800 \\ \mathrm{H} & -0.01514000 & -1.92151100 & -2.61201800 \\ \mathrm{H} & -1.41854200 & -0.96397500 & -2.14872800 \\ \mathrm{H} & -2.33210400 & -5.22463500 & -0.15390400 \\ \mathrm{O} & -2.57596800 & -4.61300700 & 4.79312000 \\ \mathrm{P} & -1.87825200 & -4.26818800 & 3.48077900 \\ \mathrm{C} & -3.21556800 & -4.23293400 & 2.21580900 \\ \mathrm{H} & -4.96560000 & -3.25021200 & 0.92399900 \\ \mathrm{H} & -3.55059900 & -5.26582000 & 2.00767000 \\ \mathrm{H} & -4.04442500 & -3.70120800 & 2.69517700 \\ \mathrm{O} & -1.31487800 & -2.82625700 & 3.46681200 \\ \mathrm{O} & -5.89040800 & -2.32349100 & 3.09306400 \\ \mathrm{P} & -5.30838700 & -0.94107400 & 2.81272200 \\ \mathrm{C} & -5.42640700 & -0.63466000 & 0.98332200 \\ \mathrm{Ac} & -1.66436600 & -0.96808000 & 1.83433700 \\ \mathrm{H} & -6.20293000 & -1.28993000 & 0.56332100 \\ \mathrm{H} & -5.80714200 & 0.38423100 & 0.87963800 \\ \mathrm{O} & -3.80058800 & -0.86054500 & 3.11860700 \\ \mathrm{H} & -4.82171000 & 0.53185600 & -1.35292900 \\ \mathrm{H} & -3.09660700 & 0.17896900 & -1.30056800 \\ \mathrm{H} & -4.63695800 & 1.93143500 & 0.67124200 \\ \mathrm{O} & -0.81752800 & -5.29196900 & 3.08551900 \\ \mathrm{H} & -3.69529000 & 2.50700000 & -0.68450200 \\ \mathrm{H} & -0.77558800 & 2.80388500 & 1.12383800 \\ \mathrm{H} & -1.83676900 & 3.43161200 & -0.12851300 \\ \mathrm{H} & -0.01255200 & 2.43206500 & -1.23723000 \\ \mathrm{H} & -1.39640200 & 1.38472200 & -1.51895300 \\ \mathrm{H} & 1.43934400 & -0.73240100 & -1.08202700 \\ \mathrm{H} & 0.70814200 & 0.36672400 & -2.25319600 \\ \mathrm{O} & -2.77995600 & 0.18229300 & 2.61866600 \\ & -6.56070000 & 3.32435800 & 3.29870000 \\ & & -2.41275200 & -0.13784600\end{array}$

\section{Ac(PETEP) $)^{8-}$}

$\begin{array}{lrrr}\mathrm{N} & -4.22746300 & -0.71936500 & 0.23942300 \\ \mathrm{C} & -3.98613000 & 0.38177400 & -0.70170600 \\ \mathrm{C} & -3.70765600 & 1.73197600 & -0.05206100 \\ \mathrm{~N} & -2.55589600 & 1.77943500 & 0.87106500 \\ \mathrm{C} & -1.41995200 & 2.48249200 & 0.26067200 \\ \mathrm{C} & -0.66085000 & 1.66230900 & -0.76853300 \\ \mathrm{~N} & 0.07545800 & 0.51914700 & -0.20727100 \\ \mathrm{C} & 0.57028500 & -0.33005600 & -1.29271000 \\ \mathrm{C} & -0.13544900 & -1.62650700 & -1.51159800 \\ \mathrm{~N} & -1.04454400 & -2.10909600 & -0.76889800 \\ \mathrm{C} & -1.55489300 & -3.43526700 & -1.11469600 \\ \mathrm{C} & -2.00838200 & -4.20073900 & 0.11945800 \\ \mathrm{~N} & -2.96120800 & -3.48995700 & 0.98151000 \\ \mathrm{C} & -4.25762500 & -3.23676800 & 0.32556200 \\ \mathrm{C} & -4.34676600 & -1.96876200 & -0.51891300\end{array}$




$\begin{array}{cccc}\mathrm{O} & -1.13688600 & 0.87837000 & 3.41683100 \\ \mathrm{P} & -1.67475000 & 2.32661600 & 3.49684500 \\ \mathrm{C} & -2.93920100 & 2.40649200 & 2.14999700 \\ \mathrm{H} & -3.57833400 & -1.95255100 & -1.29126900 \\ \mathrm{H} & -3.24195000 & 3.45975300 & 2.00653700 \\ \mathrm{H} & -3.80509700 & 1.86358000 & 2.54452100 \\ \mathrm{O} & -2.44471400 & 2.60424100 & 4.78228000 \\ \mathrm{O} & 2.92425700 & -1.16848700 & 0.58422800 \\ \mathrm{P} & 2.02191500 & -0.41903800 & 1.56392500 \\ \mathrm{C} & 1.18369500 & 0.95797200 & 0.65929300 \\ \mathrm{H} & -5.31811800 & -1.99779700 & -1.04678200 \\ \mathrm{H} & 1.93110800 & 1.53872000 & 0.08878600 \\ \mathrm{H} & 0.78291200 & 1.60780900 & 1.44262100 \\ \mathrm{O} & 0.85113100 & -1.29298700 & 2.05572400 \\ \mathrm{H} & -1.13764100 & -4.44540200 & 0.73639400 \\ \mathrm{H} & -2.38261100 & -3.32922800 & -1.82382600 \\ \mathrm{H} & -0.78717100 & -4.03217500 & -1.62580000 \\ \mathrm{H} & -4.53606700 & -4.09467300 & -0.31168200 \\ \mathrm{O} & -0.69323800 & -5.30208700 & 2.86754400 \\ \mathrm{O} & -6.05642900 & 0.18694200 & 3.59919400 \\ \mathrm{H} & -2.44120600 & -5.16220100 & -0.21363300 \\ \mathrm{O} & -2.36439800 & -4.79310700 & 4.71753100 \\ \mathrm{P} & -1.76042000 & -4.34609000 & 3.38936300 \\ \mathrm{C} & -3.18227300 & -4.27161900 & 2.21435500 \\ \mathrm{H} & -5.01013800 & -3.16487100 & 1.11698200 \\ \mathrm{H} & -3.52453700 & -5.29571800 & 1.97713400 \\ \mathrm{H} & -3.98203600 & -3.77234800 & 2.77105900 \\ \mathrm{O} & -1.26305600 & -2.88153100 & 3.42597800 \\ \mathrm{O} & -5.93671500 & -2.29665900 & 3.06692000 \\ \mathrm{P} & -5.30924800 & -0.92076600 & 2.86051300 \\ \mathrm{C} & -5.42577600 & -0.47467400 & 1.06475800 \\ \mathrm{Ac} & -1.66518500 & -0.98212300 & 1.83772300 \\ \mathrm{H} & -6.30611300 & -0.96455400 & 0.61923900 \\ \mathrm{H} & -5.64309700 & 0.59458400 & 1.06695500 \\ \mathrm{O} & -3.79499100 & -0.91279500 & 3.15117500 \\ \mathrm{H} & -4.84693400 & 0.51454000 & -1.38369700 \\ \mathrm{H} & -3.13374800 & 0.09227700 & -1.32617400 \\ \mathrm{H} & -4.59662700 & 2.07287300 & 0.48641500 \\ \mathrm{H} & -3.56747300 & 2.45690000 & -0.86762200 \\ \mathrm{H} & -0.74899300 & 2.79727100 & 1.06313000 \\ \mathrm{H} & -1.76527100 & 3.40833600 & -0.23303400 \\ \mathrm{H} & 0.03612600 & 2.33863500 & -1.29670100 \\ \mathrm{H} & -1.35221300 & 1.27912100 & -1.52740700 \\ \mathrm{H} & 1.61252700 & -0.61648500 & -1.08216800 \\ \mathrm{H} & 0.60105000 & 0.21145600 & -2.25227800 \\ \mathrm{O} & 2.79726200 & 0.24805300 & 2.69380600 \\ \mathrm{O} & -0.58222100 & 3.35361500 & 3.21873700 \\ \mathrm{H} & 0.23817200 & -2.19488100 & -2.37736400\end{array}$

Ac(PETRP) ${ }^{6-}$

$\begin{array}{lrrr}\mathrm{N} & -3.59784332 & -0.88011707 & -0.05018495 \\ \mathrm{C} & -4.25512596 & 0.18831906 & -0.23288657 \\ \mathrm{C} & -3.94623794 & 1.49908245 & 0.41188607 \\ \mathrm{~N} & -2.71971528 & 1.56827096 & 1.21365377 \\ \mathrm{C} & -1.67688546 & 2.34072288 & 0.51491408 \\ \mathrm{C} & -1.00443899 & 1.60872564 & -0.63790936 \\ \mathrm{~N} & -0.08987420 & 0.51920024 & -0.24267546 \\ \mathrm{C} & 0.35224389 & -0.20806576 & -1.44063923 \\ \mathrm{C} & -0.18209945 & -1.59160102 & -1.62500393 \\ \mathrm{~N} & -0.82956580 & -2.24318710 & -0.75040158 \\ \mathrm{C} & -1.24336437 & -3.60714825 & -1.08669028 \\ \mathrm{C} & -1.83507387 & -4.34018116 & 0.11013369 \\ \mathrm{~N} & -2.87867850 & -3.62168793 & 0.85420724 \\ \mathrm{C} & -4.08833049 & -3.30721954 & 0.08365117 \\ \mathrm{C} & -4.01147570 & -2.06032275 & -0.79593004 \\ \mathrm{O} & -1.14275560 & 0.56024498 & 3.55900390 \\ \mathrm{P} & -1.64775247 & 2.00872222 & 3.77080857 \\ \mathrm{C} & -3.00941352 & 2.15898419 & 2.53435983 \\ \mathrm{H} & -3.29910146 & -2.19391849 & -1.61266786\end{array}$




$\begin{array}{lccc}\mathrm{H} & -3.31102614 & 3.21700617 & 2.44526305 \\ \mathrm{H} & -3.85781180 & 1.61670707 & 2.96573221 \\ \mathrm{O} & -2.31208167 & 2.20205219 & 5.12707954 \\ \mathrm{O} & 2.90838340 & -0.99828960 & 0.14178042 \\ \mathrm{P} & 2.10865570 & -0.33030230 & 1.25234824 \\ \mathrm{C} & 1.06979444 & 1.01415339 & 0.52353705 \\ \mathrm{H} & -4.99847418 & -1.91440681 & -1.25830286 \\ \mathrm{H} & 1.69722854 & 1.68742057 & -0.08601021 \\ \mathrm{H} & 0.71103372 & 1.59003258 & 1.38279945 \\ \mathrm{O} & 1.05888442 & -1.28764990 & 1.87185703 \\ \mathrm{H} & -1.03260766 & -4.54322320 & 0.82731289 \\ \mathrm{H} & -1.95590646 & -3.58445495 & -1.91837020 \\ \mathrm{H} & -0.38242752 & -4.19041319 & -1.43747766 \\ \mathrm{H} & -4.39493105 & -4.15935015 & -0.54744362 \\ \mathrm{O} & -1.01709272 & -3.29353613 & 3.16505633 \\ \mathrm{O} & 2.97472613 & 0.34946851 & 2.30335104 \\ \mathrm{H} & -2.21232616 & -5.31613152 & -0.24312621 \\ \mathrm{O} & -2.75293319 & -4.30700856 & 4.79367380 \\ \mathrm{P} & -2.50220552 & -3.51017718 & 3.53401454 \\ \mathrm{C} & -3.20291142 & -4.38726159 & 2.08462264 \\ \mathrm{H} & -4.89298086 & -3.13910014 & 0.80517726 \\ \mathrm{H} & -2.82169334 & -5.41495949 & 2.02385089 \\ \mathrm{H} & -4.28716317 & -4.46200500 & 2.21393581 \\ \mathrm{O} & -3.17083483 & -2.11887573 & 3.47377634 \\ \mathrm{H} & 0.14174902 & 0.36703797 & -2.35469401 \\ \mathrm{O} & -0.57310198 & 3.03905364 & 3.45850857 \\ \mathrm{H} & 1.44483120 & -0.33257348 & -1.40927492 \\ \mathrm{Ac} & -1.43126971 & -1.12418511 & 1.77985193 \\ \mathrm{H} & -0.45522914 & 2.35722567 & -1.23515866 \\ \mathrm{H} & -1.76601071 & 1.17833804 & -1.29880759 \\ \mathrm{H} & 0.07179388 & -2.04312927 & -2.59591401 \\ \mathrm{H} & -5.13177949 & 0.20673595 & -0.89681223 \\ \mathrm{H} & -2.10893880 & 3.27085269 & 0.10803724 \\ \mathrm{H} & -4.81702820 & 1.74839309 & 1.03348866 \\ \mathrm{H} & -3.94744006 & 2.26508921 & -0.38071681 \\ \mathrm{H} & -0.93909709 & 2.64830959 & 1.26016248\end{array}$

$\begin{array}{lrrr}\mathrm{Ac}(\mathrm{PETP}) & 5- & \text { derivative } & \left(\mathrm{R}=\mathrm{CH}_{2} \mathrm{OCH}_{3}\right) \\ \mathrm{N} & -4.25164800 & -0.82600000 & 0.31530300 \\ \mathrm{C} & -4.00364600 & 0.23962700 & -0.66764700 \\ \mathrm{C} & -3.70585500 & 1.61528800 & -0.08427900 \\ \mathrm{~N} & -2.53649500 & 1.70037600 & 0.81622700 \\ \mathrm{C} & -1.38477000 & 2.32768900 & 0.14706400 \\ \mathrm{C} & -0.63649400 & 1.42533500 & -0.82268900 \\ \mathrm{~N} & 0.06254500 & 0.29288200 & -0.19472500 \\ \mathrm{C} & 0.59551300 & -0.62648700 & -1.22710300 \\ \mathrm{C} & 0.13155200 & -2.07540200 & -1.11231500 \\ \mathrm{~N} & -1.28413900 & -2.29126700 & -1.42233600 \\ \mathrm{C} & -1.69146900 & -3.68559400 & -1.21635800 \\ \mathrm{C} & -1.86602500 & -4.16645000 & 0.22092000 \\ \mathrm{~N} & -2.86345600 & -3.49147200 & 1.07349900 \\ \mathrm{C} & -4.19236900 & -3.34384600 & 0.44896700 \\ \mathrm{C} & -4.37377600 & -2.09946900 & -0.40771000 \\ \mathrm{O} & -1.13597600 & 0.94499300 & 3.39642800 \\ \mathrm{P} & -1.62562000 & 2.41437100 & 3.39067300 \\ \mathrm{C} & -2.89104400 & 2.44174000 & 2.04334400 \\ \mathrm{H} & -3.63328900 & -2.08312700 & -1.20286900 \\ \mathrm{H} & -3.15327400 & 3.49050600 & 1.81689700 \\ \mathrm{H} & -3.77551900 & 1.96428500 & 2.47827200 \\ \mathrm{O} & -2.38081300 & 2.79148100 & 4.65834300 \\ \mathrm{O} & 2.88992200 & -1.37758300 & 0.77498900 \\ \mathrm{P} & 1.95190500 & -0.57522500 & 1.67044500 \\ \mathrm{C} & 1.15320500 & 0.76288500 & 0.67974900 \\ \mathrm{H} & -5.36595300 & -2.16813600 & -0.88918400 \\ \mathrm{H} & 1.91713100 & 1.30896900 & 0.09714400 \\ \mathrm{H} & 0.74565600 & 1.45265500 & 1.42515000 \\ \mathrm{O} & 0.74738700 & -1.41208000 & 2.14913400 \\ \mathrm{H} & -0.91368200 & -4.15116200 & 0.76213800 \\ \mathrm{H} & -2.62930000 & -3.84301700 & -1.75942400\end{array}$




$\begin{array}{cccc}\mathrm{H} & -0.96547900 & -4.38399300 & -1.66939800 \\ \mathrm{H} & -4.43390700 & -4.22984500 & -0.16337300 \\ \mathrm{O} & -0.42855800 & -4.96217000 & 2.98027500 \\ \mathrm{O} & -6.20245600 & 0.06966500 & 3.64664700 \\ \mathrm{H} & -2.13548800 & -5.23540600 & 0.13392400 \\ \mathrm{O} & -2.20645000 & -4.83918200 & 4.79828400 \\ \mathrm{P} & -1.63973900 & -4.21680900 & 3.52423000 \\ \mathrm{C} & -3.02447900 & -4.27630800 & 2.31697100 \\ \mathrm{H} & -4.92246800 & -3.30407800 & 1.26229700 \\ \mathrm{H} & -3.27206900 & -5.32734100 & 2.08183000 \\ \mathrm{H} & -3.87221200 & -3.84611700 & 2.86011000 \\ \mathrm{O} & -1.38456800 & -2.70011000 & 3.67889100 \\ \mathrm{O} & -5.79759400 & -2.39430900 & 3.16572700 \\ \mathrm{P} & -5.32644700 & -0.96195400 & 2.94136800 \\ \mathrm{C} & -5.44398900 & -0.54712200 & 1.13999700 \\ \mathrm{Ac} & -1.72542400 & -0.94748400 & 1.92558500 \\ \mathrm{H} & -6.33664300 & -1.02172100 & 0.70218900 \\ \mathrm{H} & -5.63499700 & 0.52782800 & 1.13152800 \\ \mathrm{O} & -3.82510400 & -0.77790000 & 3.24979700 \\ \mathrm{H} & -4.86335800 & 0.35140200 & -1.35390300 \\ \mathrm{H} & -3.15592000 & -0.08528200 & -1.27850400 \\ \mathrm{H} & -4.58237300 & 1.98409300 & 0.45581300 \\ \mathrm{H} & -3.57691000 & 2.30198600 & -0.93425000 \\ \mathrm{H} & -0.70671300 & 2.68990000 & 0.92330300 \\ \mathrm{H} & -1.71466400 & 3.21947800 & -0.41421800 \\ \mathrm{H} & 0.07949000 & 2.05738600 & -1.37897000 \\ \mathrm{H} & -1.33450600 & 1.02454300 & -1.56543900 \\ \mathrm{H} & 1.68685300 & -0.65222000 & -1.14888100 \\ \mathrm{H} & 0.36615400 & -0.22846100 & -2.22214300 \\ \mathrm{O} & 2.68097300 & 0.13719200 & 2.80483400 \\ \mathrm{O} & -0.50162900 & 3.38346900 & 3.04639500 \\ \mathrm{H} & 0.33187600 & -2.41575400 & -0.09094400 \\ \mathrm{H} & 0.78022000 & -2.68709400 & -1.76547300 \\ \mathrm{C} & -1.64548000 & -1.84236100 & -2.77012100 \\ \mathrm{H} & -2.70784500 & -2.05635300 & -2.93471300 \\ \mathrm{H} & -1.54115600 & -0.75444200 & -2.82457800 \\ \mathrm{C} & -0.87537900 & -2.48358000 & -3.93408500 \\ \mathrm{H} & 0.18105700 & -2.17859400 & -3.93247700 \\ \mathrm{H} & -0.90739700 & -3.57452200 & -3.85104100 \\ \mathrm{O} & -1.45883000 & -2.16581200 & -5.18718600 \\ \mathrm{C} & -1.17801000 & -0.85373100 & -5.63829400 \\ \mathrm{H} & -1.65080200 & -0.08835500 & -5.00974600 \\ \mathrm{H} & -0.09467400 & -0.67082400 & -5.66407600 \\ \mathrm{H} & -1.57762500 & -0.76463900 & -6.65097600\end{array}$

Ac(PETP $)^{5-}$ derivative $2\left(\mathrm{R}=\mathrm{CHNCH}_{3}\right)$

$\begin{array}{lrrr}\mathrm{N} & -4.24098100 & -0.65553100 & 0.32021700 \\ \mathrm{C} & -4.00729400 & 0.49621200 & -0.56426800 \\ \mathrm{C} & -3.72759500 & 1.81470000 & 0.14677000 \\ \mathrm{~N} & -2.54564100 & 1.83547900 & 1.03408400 \\ \mathrm{C} & -1.40885400 & 2.51819900 & 0.39459000 \\ \mathrm{C} & -0.67109900 & 1.69223200 & -0.64945000 \\ \mathrm{~N} & 0.03979400 & 0.51984600 & -0.11372000 \\ \mathrm{C} & 0.57203400 & -0.31304100 & -1.21743800 \\ \mathrm{C} & 0.14468200 & -1.77889300 & -1.20314900 \\ \mathrm{~N} & -1.26297200 & -2.01546000 & -1.54091800 \\ \mathrm{C} & -1.65872200 & -3.42043500 & -1.37775200 \\ \mathrm{C} & -1.83145500 & -3.96287000 & 0.03583400 \\ \mathrm{~N} & -2.86241800 & -3.35699000 & 0.90012200 \\ \mathrm{C} & -4.17399000 & -3.17153200 & 0.24977100 \\ \mathrm{C} & -4.33566900 & -1.86352800 & -0.51043800 \\ \mathrm{O} & -1.15047900 & 0.85489800 & 3.56498400 \\ \mathrm{P} & -1.60839900 & 2.33206700 & 3.65610400 \\ \mathrm{C} & -2.88237800 & 2.47536900 & 2.32210600 \\ \mathrm{H} & -3.56685700 & -1.78000100 & -1.27439500 \\ \mathrm{H} & -3.14011200 & 3.54111800 & 2.18638100 \\ \mathrm{H} & -3.76733600 & 1.96956700 & 2.72356600 \\ \mathrm{O} & -2.35065300 & 2.63897800 & 4.94986800 \\ \mathrm{O} & 2.90143000 & -1.18129500 & 0.68812900\end{array}$




\begin{tabular}{|c|c|c|c|}
\hline $\mathrm{P}$ & 1.96679400 & -0.46066700 & \\
\hline G & 1.13641900 & 0.93633800 & 0.78034600 \\
\hline 11 & -5.31154000 & -1.89267600 & -1.02790800 \\
\hline & 1.88472000 & 1.53510600 & 0.23013100 \\
\hline $\mathrm{H}$ & 0.73207900 & 1.56262800 & 1.58158400 \\
\hline 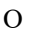 & 0.77675100 & -1.34689100 & 2.08200800 \\
\hline 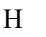 & -0.88568600 & -3.94050200 & 0.58908200 \\
\hline$\Pi$ & -2.59548500 & -3.56397700 & -1.92698000 \\
\hline & -0.92861600 & -4.09121900 & 4100 \\
\hline $\mathrm{H}$ & -4.39210100 & -4.00814900 & 6000 \\
\hline 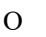 & -0.50569000 & -5.07472400 & 931100 \\
\hline$U$ & -6.20044500 & -0.04300700 & 600 \\
\hline 11 & -2.060 & -5.03579600 & 3700 \\
\hline $\mathrm{O}$ & -2.286 & -4.9 & 500 \\
\hline$P$ & -1.678 & -4.28 & 00 \\
\hline $\mathrm{C}$ & -3.0 & -4.2 & 500 \\
\hline $\mathrm{H}$ & -4.9 & -3.2 & 300 \\
\hline $\mathrm{H}$ & -3.3 & -5.2 & 800 \\
\hline $\mathrm{H}$ & -3.8 & & \\
\hline $\mathrm{O}$ & -1.334 & $-2.787 \mathrm{C}$ & 600 \\
\hline $\mathrm{O}$ & -5.824 & -2.46334 & 00 \\
\hline 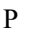 & -5.335 & -1.02359100 & 00 \\
\hline 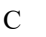 & -5.4470 & -0.46921300 & 00 \\
\hline Ac & -1.715 & 6500 & 200 \\
\hline $\mathrm{H}$ & -6.322 & -0.93083600 & 200 \\
\hline $\mathrm{H}$ & -5.66 & 0.59800000 & 00 \\
\hline $\mathrm{O}$ & -3.8 & -0.8 & 500 \\
\hline $\mathrm{H}$ & -4.87 & 0.65859 & 00 \\
\hline $\mathrm{H}$ & -3.1 & 0.2 & 00 \\
\hline $\mathrm{H}$ & & 2.107909 & \\
\hline $\mathrm{H}$ & -3.62 & 2.58590 & 00 \\
\hline $\mathrm{H}$ & -0.721 & & \\
\hline $\mathrm{H}$ & -1.75 & 3.4 & $-0 .($ \\
\hline $\mathrm{H}$ & 0.03 & 87100 & 00 \\
\hline $\mathrm{H}$ & -1.374 & 1.34340 & 00 \\
\hline $\mathrm{H}$ & 1.665 & -0.32044600 & 900 \\
\hline $\mathrm{H}$ & 0.31 & 0.15295600 & -2.1 \\
\hline $\mathrm{O}$ & 2.70 & 24500 & 2.8 \\
\hline$\Omega$ & -0.46700800 & & \\
\hline $\mathrm{H}$ & 0.34 & -2.17800 & 600 \\
\hline $\mathrm{H}$ & 0.81728400 & -2.33002300 & 3400 \\
\hline $\mathrm{C}$ & -1.603 & -1.58026000 & 600 \\
\hline $\mathrm{H}$ & -2.69 & -1.62113000 & \\
\hline $\mathrm{H}$ & -1.308 & -0.53331100 & 700 \\
\hline $\mathrm{C}$ & -0.97827400 & & 700 \\
\hline $\mathrm{H}$ & 0.11799200 & -2.48955900 & -4.01196500 \\
\hline $\mathrm{H}$ & -1.37805300 & -4.70537700 & -5.96702800 \\
\hline $\mathrm{N}$ & -1.67774000 & -2.92848100 & -4.92158200 \\
\hline $\mathrm{C}$ & -0.99766800 & -3.67768600 & -5.95873700 \\
\hline $\mathrm{H}$ & -1.23757300 & -3.24371200 & -6.93558500 \\
\hline $\mathrm{H}$ & 0.09454000 & -3.69999100 & -5.83458100 \\
\hline
\end{tabular}

$\begin{array}{lrrr}\mathrm{Ac}(\mathrm{PETP})^{5-} & \text { derivative } 3 & \left(\mathrm{R}=\mathrm{CH}_{2} \mathrm{CH}_{3}\right) \\ \mathrm{N} & -4.26185700 & -0.79206800 & 0.32212300 \\ \mathrm{C} & -4.01571900 & 0.28322000 & -0.65048200 \\ \mathrm{C} & -3.71536700 & 1.65201600 & -0.05198300 \\ \mathrm{~N} & -2.54575500 & 1.72387500 & 0.84953500 \\ \mathrm{C} & -1.39731000 & 2.36756400 & 0.19049600 \\ \mathrm{C} & -0.65524400 & 1.48603400 & -0.80327300 \\ \mathrm{~N} & 0.04644600 & 0.34002300 & -0.20308200 \\ \mathrm{C} & 0.56288000 & -0.56440100 & -1.25733200 \\ \mathrm{C} & 0.10589000 & -2.01687400 & -1.15326500 \\ \mathrm{~N} & -1.31156400 & -2.23471300 & -1.44582400 \\ \mathrm{C} & -1.70704000 & -3.63191400 & -1.24155500 \\ \mathrm{C} & -1.86913000 & -4.12351400 & 0.19342900 \\ \mathrm{~N} & -2.87081000 & -3.46407300 & 1.05346800 \\ \mathrm{C} & -4.20092700 & -3.31194200 & 0.43265300 \\ \mathrm{C} & -4.38631000 & -2.05908500 & -0.41138000 \\ \mathrm{O} & -1.13508900 & 0.92052700 & 3.41342200\end{array}$




\begin{tabular}{|c|c|c|c|}
\hline $\mathrm{P}$ & -1.63389400 & 6100 & 800 \\
\hline $\mathrm{C}$ & -2.90114700 & 2.43681800 & 2.09288400 \\
\hline $\mathrm{H}$ & -3.64852400 & -2.03479800 & -1.20870000 \\
\hline $\mathrm{H}$ & -3.16665200 & 3.48966500 & 1.88998400 \\
\hline $\mathrm{H}$ & -3.78347200 & 1.94712900 & 2.51868500 \\
\hline $\mathrm{O}$ & -2.38885900 & 2.73367700 & 4.71407600 \\
\hline $\mathrm{O}$ & 2.88887700 & -1.34696900 & 0.71395400 \\
\hline $\mathrm{P}$ & 1.95567000 & -0.56205200 & 1.62963800 \\
\hline $\mathrm{C}$ & 1.14702200 & 0.79156900 & 0.66814300 \\
\hline $\mathrm{H}$ & -5.38061200 & -2.12366100 & -0.88937100 \\
\hline $\mathrm{H}$ & 1.90644800 & 1.34582300 & 0.08709100 \\
\hline $\mathrm{H}$ & 0.74858300 & 1.47037400 & 1.42851700 \\
\hline $\mathrm{O}$ & 0.75707000 & -1.40963800 & 2.10404700 \\
\hline $\mathrm{H}$ & -0.91470200 & -4.09986500 & 0.73117100 \\
\hline $\mathrm{H}$ & -2.64796800 & -3.79378100 & -1.77821600 \\
\hline $\mathrm{H}$ & -0.97959700 & -4.32131300 & -1.70612500 \\
\hline $\mathrm{H}$ & -4.44237300 & -4.19180200 & -0.18843800 \\
\hline $\mathrm{O}$ & -0.43911000 & -4.98568700 & 88100 \\
\hline $\mathrm{O}$ & -6.17307300 & 0.06 & 20400 \\
\hline $\mathrm{H}$ & -2.12520600 & -5.19544800 & 0.10374100 \\
\hline $\mathrm{O}$ & -2.20362900 & -4.83428800 & 4.76703500 \\
\hline $\mathrm{P}$ & -1.63520700 & -4.22047800 & 3.48944100 \\
\hline $\mathrm{C}$ & -3.02805300 & -4.26002600 & 2.29016100 \\
\hline $\mathrm{H}$ & -4.92904500 & -3.2 & 2700 \\
\hline $\mathrm{H}$ & -3.28 & -5.3 & 8800 \\
\hline $\mathrm{H}$ & -3.87 & -3.8 & 9800 \\
\hline $\mathrm{O}$ & -1.35419500 & -2.70867500 & 3.64142300 \\
\hline $\mathrm{O}$ & -5.81939600 & -2.39347800 & 1900 \\
\hline $\mathrm{P}$ & -5.32301600 & -0.96627600 & 2.94783300 \\
\hline $\mathrm{C}$ & -5.44942100 & -0.52039100 & 1.15456200 \\
\hline Ac & 17200 & 47400 & 19800 \\
\hline $\mathrm{H}$ & -6.34611900 & -0.9 & 1500 \\
\hline $\mathrm{H}$ & -5.63501300 & 0.55 & 3900 \\
\hline $\mathrm{O}$ & -3.81618600 & -0.81529800 & 3.24479100 \\
\hline $\mathrm{H}$ & -4.87765300 & 0.40336300 & -1.33275800 \\
\hline $\mathrm{H}$ & -3.16972100 & -0.03572000 & -1.26703000 \\
\hline $\mathrm{H}$ & -4.59099900 & 2.01829000 & 0.49141300 \\
\hline $\mathrm{H}$ & -3.58373200 & 2.34724000 & -0.89445300 \\
\hline $\mathrm{H}$ & $-0.7-7-x$ & & 00 \\
\hline $\mathrm{H}$ & -1.73032500 & 3.27 & -0.34902500 \\
\hline $\mathrm{H}$ & 0.05791500 & 2.12889700 & -1.35087100 \\
\hline $\mathrm{H}$ & -1.35735100 & 1.10042500 & -1.55014700 \\
\hline $\mathrm{H}$ & 1.65593700 & -0.58500100 & -1.20193800 \\
\hline $\mathrm{H}$ & 0.30893100 & -0.15613900 & -2.24180800 \\
\hline $\mathrm{O}$ & 2.691 & 200 & 2.77076700 \\
\hline $\mathrm{O}$ & -0.51585900 & 900 & 3.11139800 \\
\hline $\mathrm{H}$ & 0.32441400 & -2.37021100 & -0.14001200 \\
\hline $\mathrm{H}$ & 0.74539500 & -2.61608100 & -1.82717100 \\
\hline $\mathrm{C}$ & -1.68134700 & -1.79795700 & -2.79980300 \\
\hline $\mathrm{H}$ & -2.75300400 & -1.99410000 & -2.93085200 \\
\hline $\mathrm{H}$ & -1.57017200 & -0.70941900 & -2.86696600 \\
\hline $\mathrm{C}$ & -0.93846600 & -2.43652200 & -3.97960200 \\
\hline $\mathrm{H}$ & 0.12374500 & -2.16700000 & -3.95003200 \\
\hline $\mathrm{H}$ & -0.98723800 & -3.52939500 & -3.90492000 \\
\hline $\mathrm{C}$ & -1.54266600 & -1.98587300 & -5.30754700 \\
\hline $\mathrm{H}$ & -1.00885800 & -2.42153300 & -6.15805300 \\
\hline $\mathrm{H}$ & -2.59453800 & -2.28572100 & -5.38188200 \\
\hline $\mathrm{H}$ & -1.50146700 & -0.89501600 & -5.40972300 \\
\hline
\end{tabular}




\section{REFERENCES}

1. Stein, B. W.; Morgenstern, A. L.; Batista, E. R.; Birnbaum, E. R.; Bone, S. E.; Cary, S. K.; Ferrier, M. G.; John, K. D.; Pacheco, J. L.; Kozimor, S. A.; Mocko, V.; Scott, B. L.; Yang, P., Advancing chelation chemistry for actinium and other +3 f-elements, Am, Cm, and La. J. Am. Chem. Soc. 2019, 141 (49), 19404-19414.

2. Zielinska, B.; Bilewicz, A., The hydrolysis of actinium. J. Radioanal. Nucl. Chem. 2004, 261.

3. Thiele, N. A.; Wilson, J. J., Actinium-225 for targeted $\alpha$ therapy: Coordination chemistry and current chelation approaches. Cancer Biother. Radio. 2018, 33 (8), 336-348.

4. Ziegler, T.; Rauk, A., A theoretical study of the ethylene-metal bond in complexes between $\mathrm{Cu}(1+), \operatorname{Ag}(1+), \operatorname{Au}(1+), \operatorname{Pt}(0)$ or $\mathrm{Pt}(2+)$ and ethylene, based on the Hartree-FockSlater transition-state method. Inorg. Chem. 1979, 18.

5. Bickelhaupt, F.; Baerends, E., Kohn-Sham density functional theory: Predicting and understanding chemistry. In Reviews in Computational Chemistry, Lipkowitz, K. B.; Boyd, D. B., Eds. Wiley: New York, 2007; pp 1-86.

6. Ziegler, T.; Rauk, A., Carbon monoxide, carbon monosulfide, molecular nitrogen, phosphorus trifluoride, and methyl isocyanide as sigma donors and pi acceptors. A theoretical study by the Hartree-Fock-Slater transition-state method. Inorg. Chem. 1979, 18.

7. Mitoraj, A. M. M.; Ziegler, T., A combined charge and energy decomposition scheme for bond analysis. J. Chem. Theory Comput. 2009, 5.

8. Rodríguez, J.; Bader, R.; Ayers, P.; Michel, C.; Götz, A.; Bo, C., A high performance grid-based algorithm for computing QTAIM properties. Chem. Phys. Lett. 2009, 472.

9. Rodríguez, J., An efficient method for computing the QTAIM topology of a scalar field: The electron density case. J. Comput. Chem. 2013, 34.

10. Bader, R. F. W., Atoms in Molecules: A Quantum Theory. Clarendon Press: Oxford, UK, 1990.

11. Bader, R. F. W.; Tang, T.-H.; Tal, Y.; Biegler-König, F. W., Properties of atoms and bonds in hydrocarbon molecules. J. Am. Chem. Soc. 1982, 104, 946-952.

12. Boyd, R. J.; Choi, S. C., Hydrogen bonding between nitriles and hydrogen halides and the topological properties of molecular charge distributions. Chem. Phys. Lett. 1986, 129 (1), $62-$ 65 .

13. Jacques, V.; Desreux, J. F., Complexation of thorium(IV) and uranium(IV) by a hexaacetic hexaaza macrocycle: Kinetic and thermodynamic topomers of actinide chelates with a large cavity ligand. Inorg. Chem. 1996, 35, 7205-7210. 